\title{
Inferring the genetic responses to acute drought stress across an ecological gradient
}

\author{
Jessica K. Devitt ${ }^{1 *}$, Albert Chung ${ }^{2}$ and John J. Schenk ${ }^{3}$
}

\begin{abstract}
Background: How do xerophytic species thrive in environments that experience extreme annual drought? Although critical to the survival of many species, the genetic responses to drought stress in many non-model organisms has yet to be explored. We investigated this question in Mentzelia section Bartonia (Loasaceae), which occurs throughout western North America, including arid lands. To better understand the genetic responses to drought stress among species that occur in different habitats, the gene expression levels of three species from Mentzelia were compared across a precipitation gradient. Two de novo reference transcriptomes were generated and annotated. Leaf and root tissues were collected from control and drought shocked plants and compared to one another for differential expression. A target-gene approach was also implemented to better understand how drought-related genes from model and crop species function in non-model systems.

Results: When comparing the drought-shock treatment plants to their respective control plants, we identified 165 differentially expressed clusters across all three species. Differentially expressed genes including those associated with water movement, photosynthesis, and delayed senescence. The transcriptome profiling approach was coupled with a target genes approach that measured expression of 90 genes associated with drought tolerance in model organisms. Comparing differentially expressed genes with $\mathrm{a} \geq 2 \mathrm{log}$-fold value between species and tissue types showed significant differences in drought response. In pairwise comparisons, species that occurred in drier environments differentially expressed greater genes in leaves when drought shocked than those from wetter environments, but expression in the roots mostly produced opposite results.

Conclusions: Arid-adapted species mount greater genetic responses compared to the mesophytic species, which has likely evolved in response to consistent annual drought exposure across generations. Drought responses also depended on organ type. Xerophytes, for example, mounted a larger response in leaves to downregulate photosynthesis and senescence, while mobilizing carbon and regulating water in the roots. The complexity of drought responses in Mentzelia suggest that whole organism responses need to be considered when studying drought and, in particular, the physiological mechanisms in which plants regulate water, carbon, cell death, metabolism, and secondary metabolites.
\end{abstract}

Key-words: Adaptive response, Differential gene expression, Drought, Mentzelia, Plant physiology, RNA-Seq, Transcriptome

*Correspondence: jessicadevitt10@gmail.com

1 Department of Biology, Georgia Southern University, Statesboro, GA 30460, USA

Full list of author information is available at the end of the article

\section{Background}

Land plants have been adapting to drought stress since they first evolved onto land. Despite the potentially lethal consequences of drought stress, some plants only occur in arid regions that experience severe, annual drought. To cope with drought stress, plants have evolved 
morphological, metabolic, anatomical, and physiological adaptive responses [4, 17, 41]. Understanding the molecular processes that drive physiological responses in drought-stress adaptation is crucial as Earth's climates become hotter and drier [4].

Although multiple studies have predicted ecosystem responses to instances of drought in model and agricultural species [4, 17, 28, 72, 73], non-model species have received much less attention even though they might respond differently when experiencing drought, which includes variation in the order of physiological responses, as well as the functional gene groups that differentially express in response to drought stress [28]. When drought-stress signals are received, signal transduction leads to the induction of both physiological and metabolic processes [38]. Consequently, molecular and physiological responses are usually linked, in which a change in gene expression causes a physiological change (e.g., stomatal closure). How species adapt to drought stress at the gene-expression level, however, is less well-characterized than the physiological processes they underlie, and could vary, or even converge, across plant groups.

The expression of genes associated with stress regulation plays an important role in drought response [41]. If plants regulate the impact of drought through molecular pathways, measuring the expression levels of genes that lead to physiological responses will illuminate how plants respond to stress in their natural environment. By discovering drought response genes, future studies will be able to distinguish how adaptive evolution in conjunction with physiological plasticity facilitates or constrains drought responses [14]. Although we have learned much from studies on how crop species and other model organisms respond to drought, less is known about how the remaining species of plants are able to cope with drought stress on a genetic level, especially those in xeric environments.

Plants have likely evolved numerous responses to mitigate drought stress, which includes genetic, physiological, and morphological adaptations. Environmental variation can play a further role, in which species that occur in xeric environments might respond differently to drought stress than those that occur in mesic environments. Although studies that have examined drought stress responses have shed much light on how a species responds to drought stress, experimental comparative studies of species across an environmental gradient have the potential to further tease apart how species adapt to drought stress. If we compare, for example, a group of closely related species that occur in xeric and mesic environments, hypotheses can be formulated and tested to determine how species have adapted genetic responses to drought stress.
We propose four hypotheses to explain the evolution of drought responses in a comparative framework. The first hypothesis (HA-1) proposes that xerophytes have a stronger genetic drought response than mesophytic species. If supported, we predict that xerophytes would have a significant difference in gene expression, whether up or down-regulated, whereas mesophytes would have no significant difference in gene expression. Hypothesis HA-1 suggests that the annual drought stress that xerophytes experience has selected for genetic responses and/ or selection has not favored adaptive responses at the genetic level in mesophytes.

A second hypothesis (HA-2) posits that mesophytes have a stronger response when exposed to drought conditions than xerophytes. If supported, this would mean that mesophytes have a significant difference in gene expression, whether the difference is in genes that have been up or down-regulated, whereas xerophytes shows no significant difference in gene expression. Hypothesis HA-2 suggests that morphological adaptations (e.g., reduced leaf surface area to volume ratio) in xerophytes mitigate stress caused by drought.

A third hypothesis (HA-3) proposes that all species have similarly strong genetic responses when exposed to drought conditions regardless of their environment, suggesting that species that occur across an environmental gradient have inherited genetic responses to drought from an ancestor that itself adapted to drought conditions. If supported, hypothesis HA-3 might explain why multiple species of some clades have been successful in independently evolving into xeric environments.

Our final hypothesis is a null hypothesis (Ho) which proposes that all species fail to mount a genetic response to drought, exhibiting negligible differences in gene regulation. The null hypothesis, if supported, would suggest that plants do not respond to drought at the molecular level, rather, they have other means, such as morphological adaptations, or are susceptible to the consequences of drought stress.

Non-model plant systems might provide novel responses or mechanisms not identified in other modelplant systems, which could revolutionize the way crops are genetically modified for drought tolerance, in addition to adding to the knowledge of known genes and responses associated with drought. Because of sampling bias and potential undiscovered stress responses within plants, it is important to study species that are adapted across a wide range of habitats, including xeric ecosystems.

To test the above four hypotheses, we subjected populations from three species in the genus Mentzelia L. (Loasaceae) that occur across an environmental gradient to a drought shock experiment and compared them 
to non-drought-shocked individuals. The three species of Mentzelia occur naturally across a wide environmental gradient, from the southwestern North American deserts, to mesic habitats near the Continental Divide in the Rocky Mountains [60]. Some species of Mentzelia thrive in xeric habitats, but we know very little about how they mitigate drought stress other than having morphological adaptations associated with living in xeric environments that include leaves with reduced surface area and high trichome density [18]. To determine how Mentzelia responds to acute drought stress across an environmental gradient, we subjected three species to acute drought stress and measured and compared their gene expression levels.

\section{Results}

\section{Quality assessment and annotation of assemblies}

We sequenced approximately $35 \mathrm{~Gb}$ of pair-end read data from the cDNA libraries for eight replicates of two treatments of the xerophytic $M$. filifolia, the semi-arid $M$. reverchonii, and the mesic $M$. speciosa. We sequenced $4,079,266-20,339,791$ reads per library, with an average read count of 9,004,449 per library (Fig. 1). Two replicates failed for $M$. reverchonii, one root and one leaf sample. All other sequences, except for three libraries from $M$. filifolia that produced 4,079,266, 4,099,930, and 4,800,251 reads, were above 5 million reads (Fig. 1). Average GC content for all samples of Mentzelia filifolia were 42.4\% with 112,001 genes and 214,695 transcript assemblies. Mentzelia speciosa had an average GC content of $43.5 \%$, with 143,880 genes and 259,156 transcript assemblies in total.

The quality and completeness of each reference was assessed using HISAT2 to map the original reads from each species back to the reference transcriptomes. The average alignment rate for M. filifolia was $84.7 \%$ (Table 1), and the average alignment rate for M. speciosa was $85.4 \%$ (Table 2). The Trinotate annotation report for M. filifolia showed 92,821 known sequence hits using Swiss-Prot annotation. Approximately 52,843 known GO terms were annotated along with 47,088 known pathways using KEGG pathway enrichment analysis, and 44,275 transcript annotations using EggNOG (Table 3). The Trinotate annotation report for M. speciosa showed 105,007 known sequence hits using Swiss-Prot annotation along with 60,454 GO, 53,234 KEGG, and 43,701 EggNOG

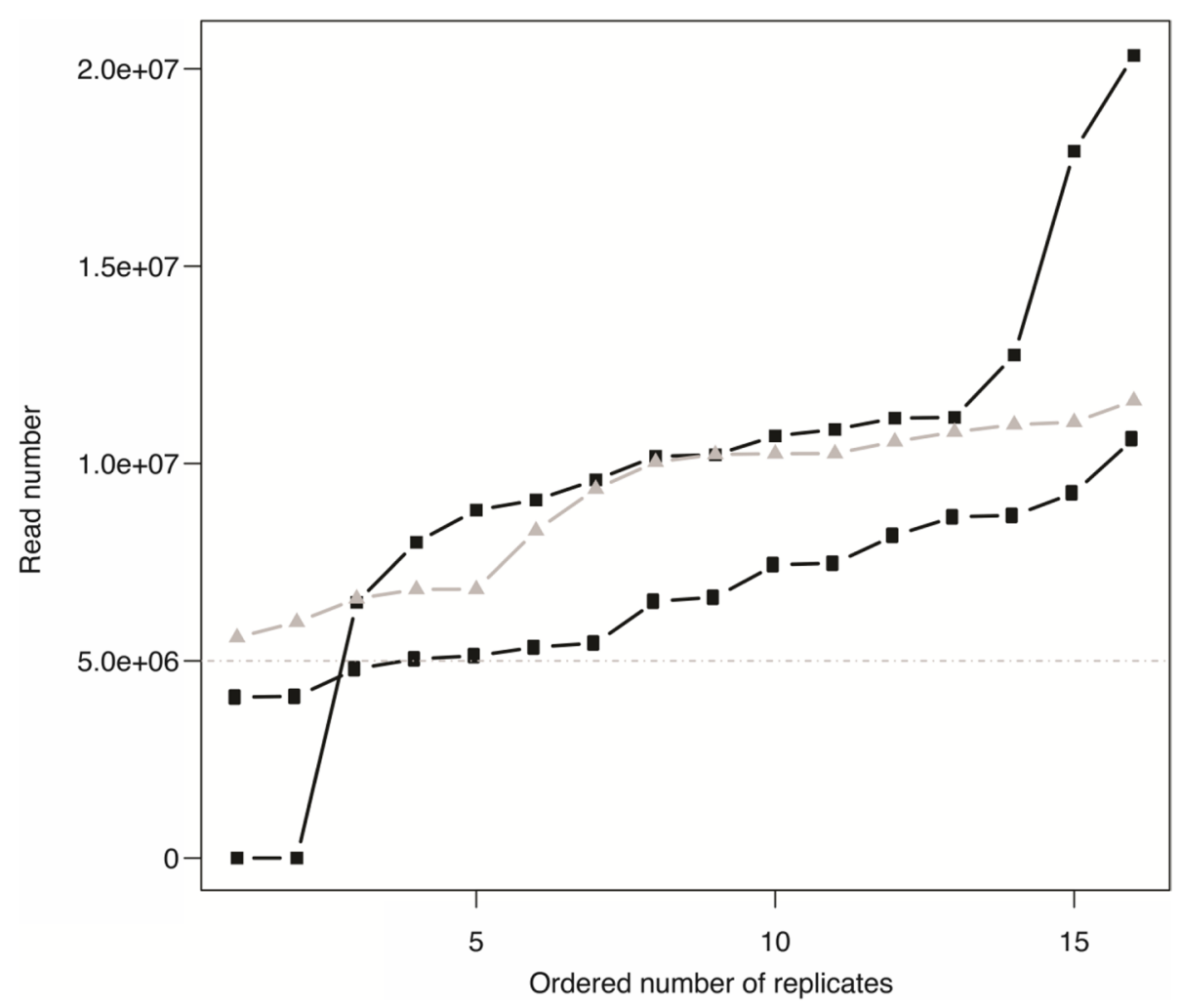

Fig. 1 Number of reads per treatment per species for Mentzelia reverchonii (black squares), M. flifolia (black circles), and M. speciosa (gray triangles). $X$-axis is ordered from the smallest to the largest number or reads per treatment. Gray horizontal dashed line at 5 million reads represents an optimal minimum number of target reads needed to infer broad differential expression patterns 
Table 1 Number of sequencing reads and alignment rate percentages calculated using HISAT2 for samples of Mentzelia filifolia. Root and leaf tissues are represented using " $\mathrm{R}$ " and " $\mathrm{L}$ " in the sample names

\begin{tabular}{lll}
\hline Sample & Number of reads & $\begin{array}{l}\text { Alignment } \\
\text { rate (\%) }\end{array}$ \\
\hline 2502_STEM & $6,375,918$ & 88.78 \\
2502_FRUIT & $6,029,059$ & 77.9 \\
2502_FLOWER & $5,857,379$ & 92.99 \\
2501_R2 & $10,627,333$ & 89.7 \\
2501_L1 & $8,175,813$ & 81.7 \\
2500_R3 & $4,800,251$ & 89.63 \\
2500_L2 & $9,254,149$ & 83.2 \\
2499_R2 & $8,682,337$ & 84.56 \\
2499_L2 & $8,647,148$ & 86.58 \\
2498_R2 & $6,510,285$ & 82.37 \\
2498_L2 & $6,612,024$ & 81.01 \\
2496_R1 & $5,348,465$ & 84.82 \\
2496_L2 & $5,128,545$ & 87.58 \\
2495_R1 & $5,048,690$ & 87.96 \\
2495_L2 & $7,435,690$ & 85.16 \\
2494_R1 & $4,099,930$ & 80.07 \\
2494_L1 & $4,079,266$ & 77.97 \\
2493_R1 & $7,471,170$ & 80.58 \\
2493_L1 & $5,450,266$ & 86.02 \\
Average: & $6,612,300.94$ & 84.66 \\
\hline
\end{tabular}

annotations. BUSCO analyses were performed to determine the completeness of each de novo assembled transcriptome using evolutionarily known gene content from universal single copy orthologs, therefore providing a measure of whether our sequencing coverage adequately sequenced transcriptomes. The transcriptomes were sufficiently sequenced, as $94.1 \%$ of the surveyed genes included in the assembly of M. filifolia were found to be complete single or duplicated genes, while $94.7 \%$ of genes surveyed from the $M$. speciosa assembly were found to be complete single or duplicated genes (Table 4).

\section{Differential expression in roots and leaves}

Among the 24 leaf and 24 root tissues sampled from three species, corset produced 64,858 leaf and 70,468 root transcript-clusters for M. filifolia, 82,205 leaf and 59,173 root transcript-clusters for $M$. reverchonii, and 54,292 leaf and 93,140 root transcript-clusters for $M$. speciosa. When considering differentially expressed (DE) genes with a log fold-change $(\operatorname{logFC}) \geq 2$, the results identified 6079 DE genes. Mentzelia filifolia leaves had 1112 DE genes, while roots had 669 DE genes. Mentzelia reverchonii had 1145 DE genes in leaves and 1411 in roots.
Table 2 Number of sequencing reads and alignment rate percentages calculated using HISAT2 for samples of Mentzelia speciosa. Root and leaf tissues are represented using " $\mathrm{R}$ " and " $\mathrm{L}$ " in the sample names

\begin{tabular}{lll}
\hline Sample & Number of reads & $\begin{array}{l}\text { Alignment } \\
\text { rate (\%) }\end{array}$ \\
\hline 001_R3 & $8,295,850$ & 86.38 \\
001_L1 & $10,983,630$ & 88.64 \\
002_R1 & $5,982,619$ & 88.39 \\
002_R3 & $10,553,058$ & 87.53 \\
003_L1 & $9,353,240$ & 88.15 \\
003_R3 & $10,251,241$ & 80.62 \\
004_L1 & $6,813,629$ & 85.69 \\
004_R3 & $6,811,305$ & 88.27 \\
006_L1 & $5,591,754$ & 73.00 \\
006_R3 & $6,575,857$ & 87.92 \\
007_L1 & $10,246,401$ & 86.77 \\
007_R3 & $10,797,019$ & 89.76 \\
008_L1 & $11,591,576$ & 85.29 \\
008_R3 & $11,046,039$ & 92.32 \\
009_L1 & $10,033,878$ & 89.23 \\
009_R2 & $10,228,942$ & 87.18 \\
011_FLOWER & $5,938,309$ & 87.66 \\
012_FRUIT & 270 & 65.74 \\
013_STEM & $7,647,140$ & 83.46 \\
Average & $8,354,829.31$ & 85.36 \\
\hline
\end{tabular}

Table 3 Annotation summary for the de novo assembly of each Mentzelia filifolia and M. speciosa

\begin{tabular}{lll}
\hline Annotation category & M. filifolia & M. speciosa \\
\hline Annotated genes & 116,537 & 122,940 \\
Transcripts with Swiss-Prot annotation & 92,821 & 105,007 \\
Transcripts with KEGG annotation & 47,088 & 53,234 \\
Transcripts with GO annotation & 52,843 & 60,454 \\
Transcripts with EggNOG annotation & 44,275 & 43,701 \\
\hline
\end{tabular}

Table 4 BUSCO results for the de novo assembled transcriptomes showing quantitative measures for each transcriptome assembly of Mentzelia filifolia and M. speciosa based on evolutionarily informed expectations of gene content

\begin{tabular}{lll}
\hline BUSCO category & M. filifolia & M. speciosa \\
\hline Complete genes & $94.10 \%$ & $94.7 \%$ \\
Complete single-copy genes & $34.70 \%$ & $26.7 \%$ \\
Complete duplicated genes & $59.40 \%$ & $68.0 \%$ \\
Fragmented genes & $4.60 \%$ & $4.60 \%$ \\
Missing & $1.30 \%$ & $0.07 \%$ \\
\hline
\end{tabular}




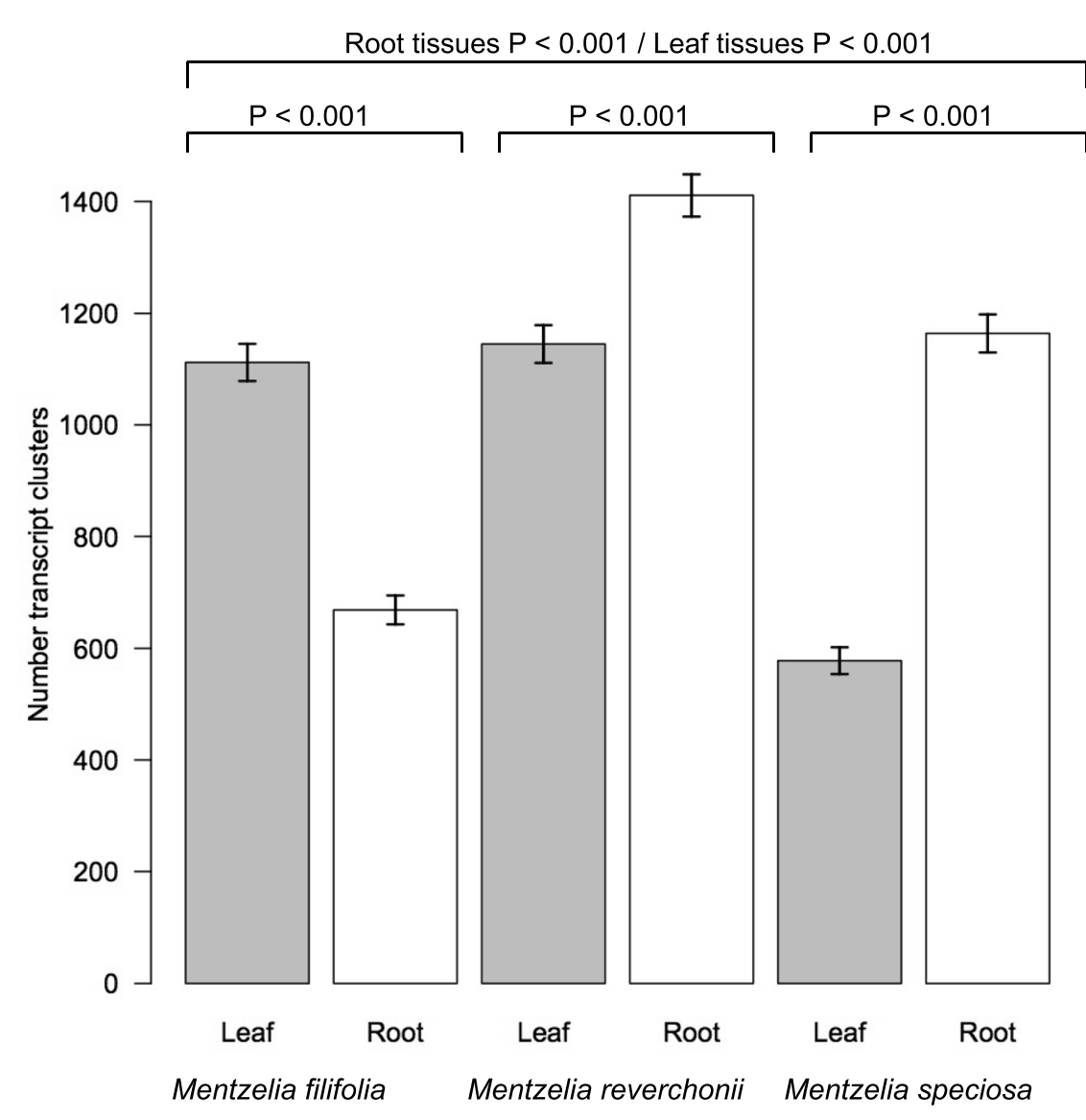

Fig. 2 Number of differentially expressed transcript clusters with a logFC $\geq 2$ from each species and tissue type resulting from the differential expression analysis in edgeR. Significant relationships between species and tissue types are designated by bars showing differences between tissues within a single species as well as differences between tissue types across species

Mentzelia speciosa resulted in 578 DE genes in leaves and 1164 in roots (Fig. 2).

The DE analyses with a false discovery rate $P$-value adjustment resulted in $165 \mathrm{DE}$ cluster-transcripts across all species and tissue types, and among them, 140 were identified to homologs (Fig. 3). The 25 remaining DE cluster transcripts were not assigned to homologous sequences and, therefore, not analyzed further. Mentzelia filifolia differentially expressed 103 transcripts in leaf and four in root tissues. Nine transcript-clusters were significantly up-regulated in $M$. filifolia leaves and 94 were significantly down-regulated (Table 5). The most downregulated genes were Adenylate cyclase proteins, which were reduced by a $\operatorname{logFC}$ of -8.4 . Up-regulated transcript-clusters were categorized as endonuclease activity, wound response, membrane components, and nitrate reductase (NADH) activity. A differential expression analysis of roots from $M$. filifolia produced four downregulated transcript-clusters (Table 6).
Mentzelia reverchonii differentially expressed two transcripts in leaf and 53 in roots. The DE analysis of leaves resulted in two over-expressed transcript-clusters, each categorized as being involved in the oxidation reduction process (Table 7). There were 28 down-regulated and 25 up-regulated transcript-clusters in the roots. The most highly up-regulated cluster was expressed with 7.0 logFC and was categorized as participating in ubiquitin-protein transferase activity and protein ubiquitination (Table 8).

Mentzelia speciosa differentially expressed three transcripts in leaf and none in root tissue. Two clusters were up-regulated and a single transcript-cluster was downregulated (Table 9 ).

The xerophytic $M$. filifolia had a stronger response than mesophytic $M$. speciosa and the intermediate semi-arid $M$. reverchonii when comparing gene response in leaves overall (Fig. 4). When comparing roots, however, $M$. speciosa and $M$. reverchonii had a greater response to drought compared to $M$. filifolia 


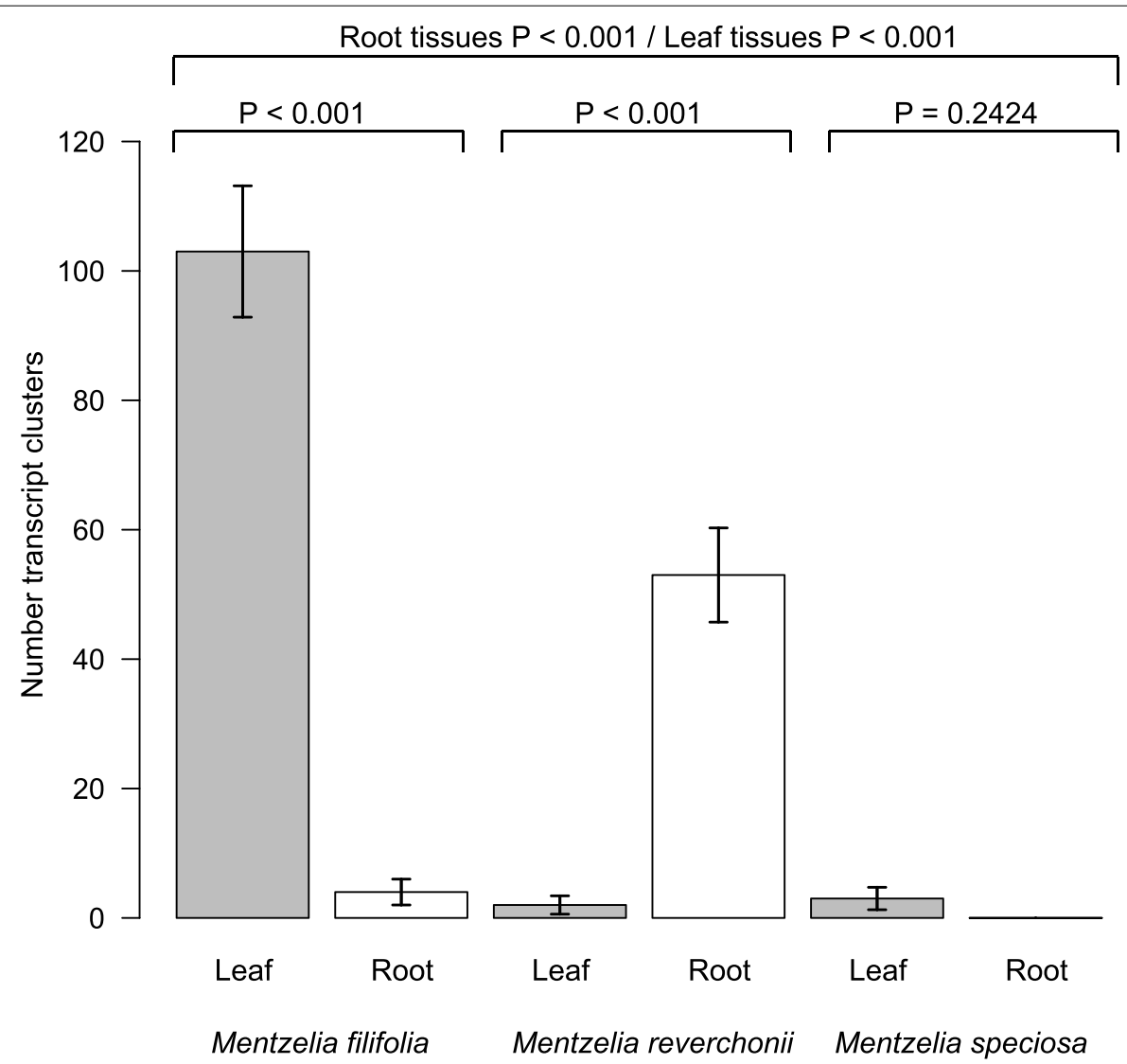

Fig. 3 Number of significant (false discovery rate $\leq 0.05$ ) differentially expressed genes from each species and tissue type resulting from the differential expression analysis using edgeR

(Fig. 4A, C). Mentzelia reverchonii produced a greater response in both leaf and root tissues when compared to M. speciosa (Fig. 4E, F), while M. filifolia produced a greater response in leaves only compared to $M$. speciosa. Overall, M. speciosa did not mount as large of a response to drought shock compared to the xeric $M$. filifolia and semi-arid M. reverchonii.

\section{Target gene expression analysis}

The target gene analysis included 90 target genes (Tables 10, 11). Because multiple transcript-clusters were identified as functions from the same process through GO annotating, multiple transcript-clusters associated with a single target gene. In total, 140 clusters matched to the targeted genes in M. filifolia leaves and 44 out of 140 clusters were included in the edgeR exact test analysis and were not filtered out before the analysis. Using a target gene approach, we compared co-expression of genes across species. From the 12 target genes that resulted in a $\operatorname{logFC} \geq 2$, we compared how they are expressed similarly between the three species and tissue types. Results are summarized in Fig. 5, Tables 2, 4, 5, 6, 7, 8, 9, 10 and 11.

\section{Discussion}

We conducted a comparative transcriptomic study of Mentzelia to understand how plants have adapted to and will respond to drought stress across an environmental gradient. The xerophytic $M$. filifolia and semi-arid $M$. reverchonii had a greater response to acute drought shock based on the number of significantly DE transcript-clusters, suggesting that species had adaptive responses in both morphological traits and genetic responses. Using results from the FDR $P$-values, we conclude that the mesophytic $M$. speciosa responded less when experiencing drought shock than the xerophytic and semi-arid adapted species, corresponding with the hypothesis that species from drought-prone environments will mount a greater genetic response than mesophytic species. Although the xerophytic and semi-arid species mounted a greater response than the mesophytic species, they showed opposite patterns in the tissues that responded. The genetic response to drought shock in M. filifolia 
Table 5 Differentially expressed genes in leaf tissue of Mentzelia filifolia from the drought-shocked treatment compared to the control. Measures of log-fold change (logFC) and false discovery rate (FDR) were used to determine significance and direction of regulation. LogFC values are in comparison to the control levels of expression from the same tissue/species

\begin{tabular}{|c|c|c|c|}
\hline Transcript cluster ID & Annotation & $\log \mathrm{FC}$ & FDR \\
\hline Cluster-58816.0 & PREDICTED: uncharacterized protein LOC104428360 & -8.37 & 0.0028 \\
\hline Cluster-48670.0 & Protein TAR1 & -7.81 & 0.0013 \\
\hline Cluster-58105.0 & Hypothetical protein CDL37_04775, partial & -7.72 & 0.0011 \\
\hline Cluster-39297.0 & Hypothetical protein CDL37_04775, partial & -7.60 & 0.0011 \\
\hline Cluster-50655.1 & Hypothetical protein T12_6945, partial & -7.59 & 0.0011 \\
\hline Cluster-41545.0 & PREDICTED: uncharacterized protein LOC109358019 & -7.52 & 0.0011 \\
\hline Cluster-60839.0 & Protein TAR1 & -7.49 & 0.0011 \\
\hline Cluster-34501.0 & Hypothetical protein BC332_34878 & -7.49 & 0.0011 \\
\hline Cluster-62161.0 & Predicted protein & -7.44 & 0.0015 \\
\hline Cluster-34654.0 & Protein TAR1 & -7.40 & 0.0011 \\
\hline Cluster-7656.2 & Transmembrane protein, putative & -7.38 & 0.0011 \\
\hline Cluster-41578.0 & Hypothetical protein BC332_34878 & -7.36 & 0.0011 \\
\hline Cluster-41545.1 & PREDICTED: uncharacterized protein LOC106795708 & -7.35 & 0.0011 \\
\hline Cluster-50091.0 & Protein TAR1 & -7.30 & 0.0011 \\
\hline Cluster-49212.0 & ATP synthase subunit beta & -7.29 & 0.0011 \\
\hline Cluster-31834.0 & Protein TAR1 & -7.28 & 0.0024 \\
\hline Cluster-49252.0 & Hypothetical protein GLYMA_13G0132002, partial & -7.23 & 0.0054 \\
\hline Cluster-34739.0 & Predicted protein & -7.22 & 0.0013 \\
\hline Cluster-36863.0 & Regulator of rDNA transcription protein 15 & -7.20 & 0.0011 \\
\hline Cluster-47860.0 & Protein TAR1 & -7.19 & 0.0011 \\
\hline Cluster-40814.0 & Protein TAR1 & -7.19 & 0.0018 \\
\hline Cluster-52445.0 & Regulator of rDNA transcription protein 15 & -7.19 & 0.0011 \\
\hline Cluster-35862.0 & Uncharacterized protein LOC110824766, partial & -7.18 & 0.0018 \\
\hline Cluster-51124.0 & Alpha-L1 nicotinic acetyl choline receptor & -7.14 & 0.0011 \\
\hline Cluster-59385.0 & Hypothetical protein CQW23_33511 & -7.14 & 0.0011 \\
\hline Cluster-37035.0 & Cytochrome P450 monooxygenase & -7.12 & 0.0015 \\
\hline Cluster-57663.0 & Predicted protein & -7.11 & 0.0011 \\
\hline Cluster-51272.0 & Regulator of rDNA transcription protein 15 & -7.10 & 0.0011 \\
\hline Cluster-60616.1 & Hypothetical protein CQW23_33755 & -7.08 & 0.0018 \\
\hline Cluster-59060.0 & Predicted protein & -7.02 & 0.0011 \\
\hline Cluster-52045.0 & Cytochrome P450 like_TBP & -7.01 & 0.0011 \\
\hline Cluster-23459.2 & Regulator of rDNA transcription protein 15 & -7.00 & 0.0011 \\
\hline Cluster-42269.0 & Protein TAR1 & -6.97 & 0.0011 \\
\hline Cluster-62261.0 & Predicted protein & -6.93 & 0.0011 \\
\hline Cluster-36057.0 & CASP-like protein $4 \mathrm{~A} 3$ & -6.91 & 0.0011 \\
\hline Cluster-38658.0 & Protein TAR1 & -6.87 & 0.0011 \\
\hline Cluster-59522.0 & Transmembrane protein, putative & -6.84 & 0.0011 \\
\hline Cluster-63249.0 & Aquaporin TIP1-1 & -6.52 & 0.0011 \\
\hline Cluster-55831.0 & Protein TAR1-like & -6.52 & 0.0016 \\
\hline Cluster-41007.0 & Regulator of rDNA transcription protein 15 & -6.47 & 0.0021 \\
\hline Cluster-51769.0 & Regulator of rDNA transcription protein 15 & -6.37 & 0.0016 \\
\hline Cluster-56791.0 & Uncharacterized protein LOC109940280 & -6.37 & 0.0032 \\
\hline Cluster-50338.0 & Hypothetical protein BUMB_02141 & -6.31 & 0.0023 \\
\hline Cluster-31006.0 & Regulator of rDNA transcription protein 15 & -6.24 & 0.0012 \\
\hline Cluster-59353.0 & Regulator of rDNA transcription protein 15 & -6.06 & 0.0018 \\
\hline Cluster-50764.0 & Hypothetical protein T459_27227 & -6.04 & 0.0051 \\
\hline Cluster-51946.0 & Regulator of rDNA transcription protein 15 & -6.04 & 0.0034 \\
\hline Cluster-60097.0 & Regulator of rDNA transcription protein 15 & -5.89 & 0.0029 \\
\hline
\end{tabular}


Table 5 (continued)

\begin{tabular}{|c|c|c|c|}
\hline Transcript cluster ID & Annotation & $\log \mathrm{FC}$ & FDR \\
\hline Cluster-42721.0 & Regulator of rDNA transcription protein 15 & -5.87 & 0.0028 \\
\hline Cluster-56732.0 & No homology & -5.72 & 0.0067 \\
\hline Cluster-35355.0 & Cytochrome P450-like TBP protein & -5.59 & 0.0137 \\
\hline Cluster-46825.0 & Regulator of rDNA transcription protein 15 & -5.57 & 0.0032 \\
\hline Cluster-53270.0 & Hypothetical protein CQW23_33511 & -5.40 & 0.0052 \\
\hline Cluster-44046.0 & Probable inactive patatin-like protein 9 & -5.06 & 0.0054 \\
\hline Cluster-32710.0 & PREDICTED: uncharacterized protein LOC104811909 & -5.04 & 0.0149 \\
\hline Cluster-34807.0 & Zinc finger protein 1 & -4.99 & 0.0189 \\
\hline Cluster-59670.0 & Senescence-associated protein, putative & -4.97 & 0.0193 \\
\hline Cluster-41530.0 & Hypothetical protein SERLA73DRAFT_67532, partial & -4.93 & 0.0011 \\
\hline Cluster-51948.0 & Hypothetical protein GLYMA_13G013900 & -4.91 & 0.0016 \\
\hline Cluster-61489.1 & Predicted protein & -4.80 & 0.0016 \\
\hline Cluster-53940.0 & Wound-responsive family protein & -4.79 & 0.0331 \\
\hline Cluster-57103.0 & Delta(24)-sterol reductase & -4.73 & 0.0185 \\
\hline Cluster-31264.0 & Hypothetical protein OXYTRI_14248 (macronuclear) & -4.70 & 0.0016 \\
\hline Cluster-61489.2 & Hypothetical protein GLYMA_13G015500, partial & -4.65 & 0.0018 \\
\hline Cluster-60530.0 & Probable inactive receptor kinase At5g67200 & -4.55 & 0.0365 \\
\hline Cluster-38796.0 & Uncharacterized protein LOC110277292 & -4.54 & 0.0025 \\
\hline Cluster-62327.0 & Uncharacterised protein & -4.47 & 0.0388 \\
\hline Cluster-61719.0 & Organ-specific protein S2-like & -4.43 & 0.0445 \\
\hline Cluster-53551.0 & Tar1p like protein & -4.38 & 0.0022 \\
\hline Cluster-55762.0 & Tar1p, partial & -4.35 & 0.0140 \\
\hline Cluster-42910.0 & PREDICTED: uncharacterized protein LOC105736981 & -4.33 & 0.0031 \\
\hline Cluster-62007.0 & Hypothetical protein GLYMA_U007300 & -4.27 & 0.0113 \\
\hline Cluster-35209.0 & No homology & -4.23 & 0.0194 \\
\hline Cluster-42867.0 & Acidic endochitinase-like & -4.22 & 0.0462 \\
\hline Cluster-50876.0 & Indole-3-acetic acid-induced protein ARG2-like & -4.16 & 0.0392 \\
\hline Cluster-48507.0 & Regulator of rDNA transcription protein 15 & -4.15 & 0.0392 \\
\hline Cluster-57366.0 & No homology & -4.10 & 0.0053 \\
\hline Cluster-52390.3 & Metallocarboxypeptidase inhibitor & -4.07 & 0.0123 \\
\hline Cluster-63044.0 & Hypothetical protein GOBAR_DD19277 & -4.05 & 0.0251 \\
\hline Cluster-54706.0 & S-adenosylmethionine decarboxylase proenzyme-like & -3.99 & 0.0270 \\
\hline Cluster-36407.0 & Hypothetical protein GLYMA_U007300 & -3.96 & 0.0093 \\
\hline Cluster-59427.0 & Hypothetical protein SERLA73DRAFT_67532, partial & -3.91 & 0.0073 \\
\hline Cluster-41436.2 & Chromosome 3B, genomic scaffold & -3.89 & 0.0117 \\
\hline Cluster-61466.0 & rRNA intron-encoded homing endonuclease, putative & -3.86 & 0.0251 \\
\hline Cluster-61875.0 & rRNA intron-encoded homing endonuclease & -3.86 & 0.0194 \\
\hline Cluster-51170.0 & UNKNOWN & -3.59 & 0.0295 \\
\hline Cluster-34949.0 & Indole-3-acetate O-methyltransferase 1 & -3.56 & 0.0270 \\
\hline Cluster-59063.0 & PREDICTED: uncharacterized protein LOC109176672 & -3.54 & 0.0178 \\
\hline Cluster-42283.0 & Senescence-associated protein & -3.48 & 0.0416 \\
\hline Cluster-34872.0 & Cold-regulated protein & -3.37 & 0.0270 \\
\hline Cluster-43131.0 & Tonoplast dicarboxylate transporter & -3.27 & 0.0445 \\
\hline Cluster-34593.0 & No homology & -3.21 & 0.0331 \\
\hline Cluster-54555.0 & No homology & -2.94 & 0.0404 \\
\hline Cluster-47071.0 & Hypothetical protein ABT39_MTgene6262 & 2.98 & 0.0451 \\
\hline Cluster-61726.0 & No homology & 3.25 & 0.0138 \\
\hline Cluster-40722.0 & No homology & 3.78 & 0.0335 \\
\hline Cluster-62217.0 & Protein RADIALIS-like 5 & 3.98 & 0.0375 \\
\hline
\end{tabular}


Table 5 (continued)

\begin{tabular}{|c|c|c|c|}
\hline Transcript cluster ID & Annotation & $\log \mathrm{FC}$ & FDR \\
\hline Cluster-52889.0 & Nitrate reductase 2 & 4.13 & 0.0331 \\
\hline Cluster-33868.0 & Crocetin glucosyltransferase, chloroplastic-like & 4.32 & 0.0171 \\
\hline Cluster-63829.0 & No homology & 6.40 & 0.0024 \\
\hline Cluster-48746.0 & No homology & 7.00 & 0.0025 \\
\hline Cluster-33695.0 & Probable xyloglucan endotransglucosylase/Hydrolase protein 23 & -4.54 & 0.0080 \\
\hline Cluster-58851.0 & Pentatricopeptide repeat-containing protein At1g07590, Mitochondrial & 3.64 & 0.0134 \\
\hline
\end{tabular}

Table 6 Differentially expressed genes in root tissue of Mentzelia. filifolia from the drought-shocked treatment compared to the control. Measures of the log-fold change (logFC) and false discovery rate (FDR) were used to determine significance and direction of regulation

\begin{tabular}{llll}
\hline Transcript cluster ID & Annotation & logFC & FDR \\
\hline Cluster-33699.0 & $\begin{array}{l}\text { Transcript antisense to } \\
\text { ribosomal RNA protein }\end{array}$ & 9.99 & 0.0153 \\
Cluster-65674.0 & CYP76A26-like protein & -4.92 & 0.0153 \\
Cluster-60975.0 & No homology & 8.08 & 0.0153 \\
Cluster-49634.0 & No homology & 7.23 & 0.0164 \\
\hline
\end{tabular}

Table 7 Differentially expressed genes in leaf tissue of Mentzelia reverchonii from the drought-shocked treatment compared to the control. Measures of the log-fold change (logFC) and false discovery rate (FDR) were used to determine significance and direction of regulation

\begin{tabular}{llll}
\hline Transcript cluster ID & Annotation & logFC & FDR \\
\hline Cluster-49973.1 & $\begin{array}{l}\text { Ribulose bisphos- } \\
\text { phate carboxylase } \\
\text { small chain }\end{array}$ & 8.98 & 0.0154 \\
Cluster-24541.01 & $\begin{array}{l}\text { Ong-chain-alcohol } \\
\text { oxidase FAO2-like }\end{array}$ & 4.25 & 0.0167 \\
\hline
\end{tabular}

was greater in the leaf than the root tissue, whereas the genetic response in $M$. reverchonii was more apparent in roots than leaves. Each species could have a different reaction time to drought stress, which could have been affected by individual plant size [15]. Plant age affects water and carbon availability, resulting in younger and smaller plants being more susceptible to drought shock [15]. The differences in response in tissues might suggest that the cascade of physiological events within leaves and roots are different between species.

Delayed senescence was differentially expressed in leaves and roots of M. filifolia and M. reverchonii. Mechanisms associated with delayed senescence, such as jasmonic acid production, auxin regulation, and Serine/ threonine-protein kinase CHK1-like protein, were identified among the DE transcript-clusters. Delaying cell death might be a response that has evolved through numerous generations of drought exposure. The historical severity and length of drought experienced by each species could be a factor that determines whether delayed senescence is a utilized pathway. Risking embolism and desiccation in order to delay senescence is an interesting process that should be further studied in xerophytes because it seems to play a major role in their adaptive response drought stress.

The three species in our experiment mounted similar, but not identical, genetic responses when exposed to drought. Many of the selected target genes were not found in our data set, however, because of filtering parameters and the large number of comparisons made during the statistical analysis, many could have been removed from the final dataset. Many target genes experienced greater than two $\log \mathrm{FC}$, which is biologically significant and points to common molecular change that occur in response to stress across distantly related species. Although few target genes resulted in significant changes, we determined that genes that play a role in drought tolerance in other plant species are also acting within the plant systems of Mentzelia.

The mesophytic M. speciosa exhibited a genetic response to drought, but because it is exposed to drought stress less often, it might have responded less quickly to stress, suggesting a less efficient and effective response when compared to species in drier environments. If the response was immediate but subsided quickly, the time of tissue collection could have missed the point with highest differential expression. Alternately, if the mechanisms triggered by drought in $M$. speciosa was delayed, the time of tissue collection could have occurred before the genetic response.

Our sequencing depth was adequate to produce transcriptomes that measured large responses to acute drought-shock. More subtle genetic responses that had lower expression levels were unlikely to have been detected by our sequencing coverage, but could still play an important role in how plants respond to acute 
Table 8 Differentially expressed genes in root tissue from Mentzelia reverchonii from the drought-shocked treatment compared to the control. Measures of the log-fold change (logFC) and false discovery rate (FDR) were used to determine significance and direction of regulation

\begin{tabular}{|c|c|c|c|}
\hline Transcript cluster ID & Annotation & $\log \mathrm{FC}$ & FDR \\
\hline Cluster-38908.0 & Remorin-like isoform X2 & 6.30 & 0.0152 \\
\hline Cluster-49883.0 & NAC domain-containing protein 72 & 4.75 & 0.0152 \\
\hline Cluster-55672.0 & GATA transcription factor 8-like & 4.33 & 0.0152 \\
\hline Cluster-18781.0 & $21 \mathrm{kDa}$ protein-like & 6.65 & 0.0223 \\
\hline Cluster-36496.0 & Non-specific lipid transfer protein GPI-anchored 2-like & 4.45 & 0.0224 \\
\hline Cluster-35195.0 & PREDICTED: uncharacterized protein LOC108988160 & -4.56 & 0.0224 \\
\hline Cluster-51946.0 & No homology & -4.31 & 0.0224 \\
\hline Cluster-25227.0 & U-box domain-containing protein 19 & 7.00 & 0.0224 \\
\hline Cluster-27607.0 & Squamosa promoter-binding protein 1-like & 4.65 & 0.0224 \\
\hline Cluster-35437.0 & CHK1 checkpoint-like protein & -10.93 & 0.0224 \\
\hline Cluster-56684.0 & No homology & -4.69 & 0.0224 \\
\hline Cluster-15975.0 & PREDICTED: uncharacterized protein LOC100854478 & 3.61 & 0.0224 \\
\hline Cluster-20450.0 & REF/SRPP-like protein At3g05500 & 5.96 & 0.0224 \\
\hline Cluster-41375.0 & Cellulose synthase A catalytic subunit 2 & 5.04 & 0.0224 \\
\hline Cluster-16220.0 & No homology & 5.34 & 0.0224 \\
\hline Cluster-47851.0 & No homology & 3.78 & 0.0289 \\
\hline Cluster-17790.0 & Acyl-lipid (9-3)-desaturase-like & 3.77 & 0.0289 \\
\hline Cluster-51094.0 & Unknown & -5.75 & 0.0289 \\
\hline Cluster-57461.0 & Ubiquitin-like protein & 3.35 & 0.0320 \\
\hline Cluster-25176.0 & Pollen-specific protein SF21-like & 3.72 & 0.0320 \\
\hline Cluster-38124.0 & L-ascorbate oxidase homolog & 5.81 & 0.0320 \\
\hline Cluster-34320.0 & No homology & -4.14 & 0.0331 \\
\hline Cluster-49004.0 & No homology & 4.83 & 0.0347 \\
\hline Cluster-30840.0 & Two-pore potassium channel 1-like & 4.93 & 0.0347 \\
\hline Cluster-20850.0 & Sodium/calcium exchanger NCL-like & 5.30 & 0.0347 \\
\hline Cluster-37384.0 & Sodium/calcium exchanger NCL-like & 3.91 & 0.0357 \\
\hline Cluster-48594.0 & Atp synthase subunit beta & -7.03 & 0.0362 \\
\hline Cluster-11814.0 & No homology & -3.32 & 0.0362 \\
\hline Cluster-39132.0 & Inositol-tetrakisphosphate 1-kinase 1-like & 4.30 & 0.0381 \\
\hline Cluster-38020.0 & No homology & -3.35 & 0.0440 \\
\hline Cluster-47551.0 & $10 \mathrm{kDa}$ putative secreted protein & -6.65 & 0.0440 \\
\hline Cluster-55259.0 & No homology & -3.92 & 0.0440 \\
\hline Cluster-33758.0 & Hypothetical protein X975_24482, partial & -7.16 & 0.0453 \\
\hline Cluster-25182.0 & Glucose repressible protein Grg1 & -5.13 & 0.0453 \\
\hline Cluster-23747.0 & Predicted protein & -7.41 & 0.0453 \\
\hline Cluster-23745.0 & Probable WRKY transcription factor 75 & -3.18 & 0.0453 \\
\hline Cluster-24748.0 & Protein SRC2-like & 3.81 & 0.0453 \\
\hline Cluster-51512.0 & Predicted protein & -6.41 & 0.0453 \\
\hline Cluster-5078.0 & Late embryogenesis abundant protein & 4.99 & 0.0453 \\
\hline Cluster-53464.0 & Putative oRF58e & -9.37 & 0.0453 \\
\hline Cluster-41067.0 & UNKNOWN & -7.99 & 0.0453 \\
\hline Cluster-7120.0 & Hypothetical protein V565_194550, partial & -10.29 & 0.0467 \\
\hline Cluster-17962.0 & No homology & -8.68 & 0.0467 \\
\hline Cluster-27793.0 & Homeobox protein $\mathrm{SBH} 1$ & -5.01 & 0.0467 \\
\hline Cluster-23243.0 & 17.3 kDa class I heat shock protein-like & 5.14 & 0.0475 \\
\hline Cluster-22949.0 & PG1 protein, homology to Homo sapiens & -8.80 & 0.0477 \\
\hline Cluster-56300.0 & No homology & -5.63 & 0.0488 \\
\hline Cluster-24206.0 & Extradiol ring-cleavage dioxygenase-like & -2.75 & 0.0494 \\
\hline
\end{tabular}


Table 8 (continued)

\begin{tabular}{llrr}
\hline Transcript cluster ID & Annotation & logFC & FDR \\
\hline Cluster-33282.0 & No homology & -3.41 & 0.0496 \\
Cluster-36817.1 & No homology & -3.60 & 0.0496 \\
Cluster-16449.0 & Uncharacterised protein & -8.93 & 0.0496 \\
Cluster-42928.0 & Uncharacterized aarF domain-containing protein kinase At1g79600, chloro- & -3.68 & 0.0224 \\
& plastic & 6.05 & 0.0374 \\
Cluster-36934.0 & Sec-independent protein translocase protein TATC, Chloroplastic & & \\
\hline
\end{tabular}

Table 9 Differentially expressed genes in leaf tissue from Mentzelia speciosa from the drought-shocked treatment compared to the control. Measures of the log-fold change $(\log F C)$ and false discovery rate (FDR) were used to determine significance and direction of regulation

\begin{tabular}{llll}
\hline Transcript cluster ID & Annotation & logFC & FDR \\
\hline Cluster-38,359.0 & Polyubiquitin-like & 7.25 & 0.0141 \\
Cluster-44,349.15 & Glucose repressible protein Grg1 & 8.49 & 0.0396 \\
Cluster-27,554.0 & Uncharacterized protein & -4.60 & 0.0396 \\
\hline
\end{tabular}

drought. Future studies that sequence at greater depths are likely to determine the low expression responses that plants mount as they respond to drought.

\section{Differentially expressed transcript-clusters in leaves}

The greatest response to drought tolerance occurred in the leaves M. filifolia (Table 5), which is unsurprising given that leaves are the main site of evapotranspiration and would be the first organs to senesce under drought. The large response of down-regulated transcript-clusters implies that metabolic processes are being downregulated to conserve water and prevent tissue and cell damage. Transcript-clusters were categorized as transmembrane associated proteins, nucleic acid binding proteins, auxin regulation, rDNA transcription, endonuclease activity, or other enzymatic activities. Four transcript-clusters were identified as proteins involved in stress response, senescence, and wound response. Suppressing drought induced leaf senescence greatly increases drought tolerance in transgenic lines of Nicotiana tabacum [55], and M. filifolia also appears to be down-regulating senescence-associated proteins. Rivero et al. [55] determined that delaying senescence in transgenic lines of $N$. tabacum increased processes of reactive oxygen species scavenging, leading to extra protection for the photosynthetic apparatus that increased water use efficiency under drought stress. Jasmonic acid, a phytohormone responsible for signal transfer in response to senescence [37], was significantly down-regulated in M. filifolia. Down-regulating jasmonic acid, a known senescence accelerator [35], would delay senescence, which appears to be a large component of how $M$. filifolia mitigates drought stress damage in leaves.

Transcription-factor proteins associated with auxin production and transport were down-regulated. Auxin response factors are associated with drought responses because of their role in hormonal response signaling as well as developmental and senescence processes [54]. When exposed to drought stress, developmental processes would most likely stop, inducing down-regulation of auxin. A single auxin related protein was upregulated in leaves, suggesting a dynamic response in the role of auxin during acute drought stress in Mentzelia. Ke et al. [35] showed that when a transgenic line of poplar was designed to overproduce auxin, drought stress tolerance was increased. Auxin metabolism is monitored by many other metabolic pathways and plays a large role in overall plant homeostasis [35]. Similar to the down-regulation of senescence, auxin regulation might be delayed to prevent the occurrence of senescence related processes, but a single occurrence of upregulation could be attributed to its role as a hormonal response signal or other developmental processes. Overall, M. filifolia leaves have a large response of down-regulation involved with senescence brought on by drought stress, and general metabolic processes, like

(See figure on next page.)

Fig. 4 Pairwise comparisons of differentially expressed genes in the drought-shock treatment among the tissue types of the focal species. Each point represents the absolute expression level of a gene/cluster for both species being compared. The dashed black line is the expected line $($ slope $=1)$ if the two species expressed all loci at identical levels and informs whether one species/tissue is mounting a greater response than the other. The solid black line is the best fit line for the data given a linear model and tells us where the majority of the points lay. All solid black slopes were significantly different than 1 at alpha $=0.05(P<0.001)$. The grey open circles indicate a log-fold change less than 2 , and the solid black circles indicate a log-fold change of 2 or greater. In all comparisons, the species that occurs in the more arid habitat is placed on the $y$-axis 

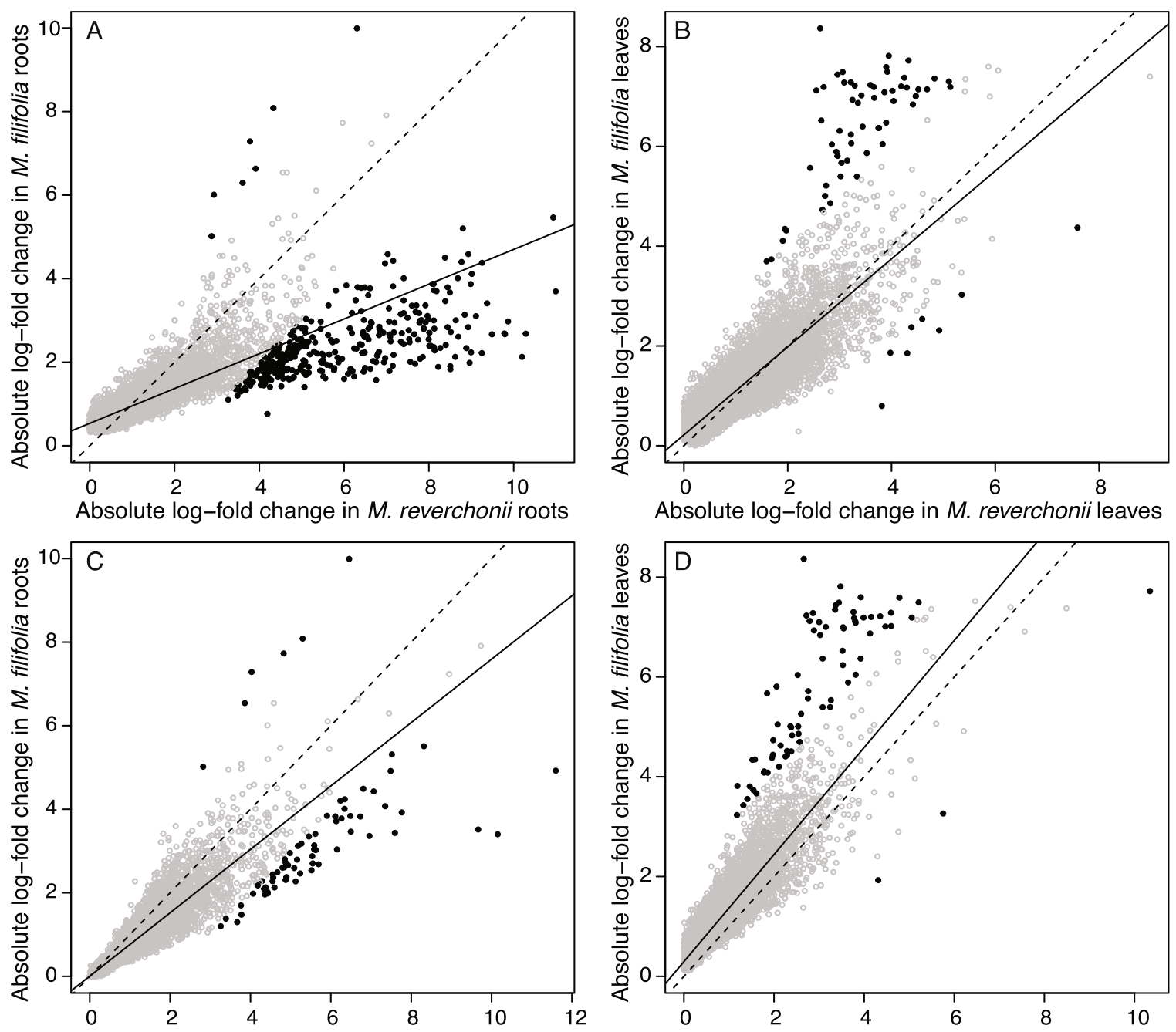

Absolute log-fold change in $M$. speciosa roots

Absolute log-fold change in $M$. speciosa leaves
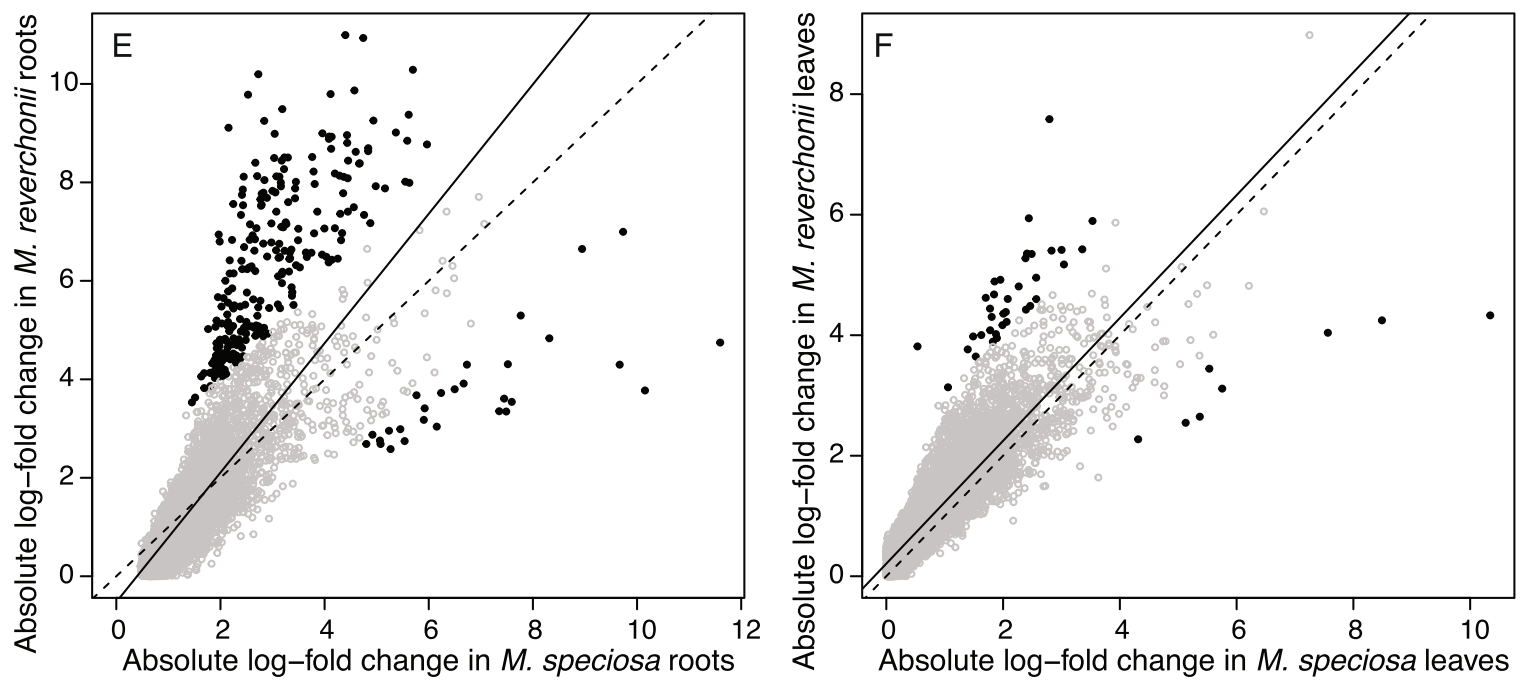

Fig. 4 (See legend on previous page.) 
Table 10 Set of target genes selected from previous studies that were determined to respond to drought

\begin{tabular}{|c|c|c|}
\hline Entry & Entry name & Protein name \\
\hline Q6ZKC0 & 14333_ORYSJ & 14-3-3-like protein GF14-C \\
\hline Q9SGW3 & PSD8A_ARATH & 265 proteasome non-ATPase regulatory subunit \\
\hline Q9SIB2 & KCS12_ARATH & 3-ketoacyl-CoA synthase 12 \\
\hline Q9LRR7 & NCED3_ARATH & 9-cis-epoxycarotenoid dioxygenase NCED3 \\
\hline Q9FH76 & ABAH3_ARATH & Abscisic acid 8'-hydroxylase 3 \\
\hline O80920 & PYL4_ARATH & Abscisic acid receptor PYL4 \\
\hline Q9FLB1 & PYL5_ARATH & Abscisic acid receptor PYL5 \\
\hline Q9FGM1 & PYL8_ARATH & Abscisic acid receptor PYL8 \\
\hline Q9M7Q4 & AI5L5_ARATH & ABSCISIC ACID-INSENSITIVE 5-like protein 5 \\
\hline Q9FXT4 & AGAL_ORYSJ & Alpha-galactosidase \\
\hline Q39958 & Q39958_HELAN & Aquaporin \\
\hline P43286 & PIP21_ARATH & Aquaporin PIP2-1 \\
\hline Q9SI64 & SPE1_ARATH & Arginine decarboxylase 1 \\
\hline Q9FT74 & RQL1_ARATH & ATP-dependent DNA helicase Q-like 1 \\
\hline QoWVW7 & RQL5_ARATH & ATP-dependent DNA helicase Q-like 5 \\
\hline Q10D00 & SUV3M_ORYSJ & ATP-dependent RNA helicase SUV3 \\
\hline P93022 & ARFG_ARATH & Auxin response factor 7 \\
\hline Q9SRU2 & BIG_ARATH & Auxin transport protein $\mathrm{BIG}$ \\
\hline Q96247 & AUX1_ARATH & Auxin transporter protein 1 \\
\hline Q6IVL3 & Q6IVL3_GOSHI & C-repeat binding factor 15 \\
\hline Q9M101 & CDPKN_ARATH & Calcium-dependent protein kinase 23 \\
\hline Q9S7J7 & CB22_ARATH & Chlorophyll a-b binding protein 2.2 \\
\hline Q9S7M0 & CB3_ARATH & Chlorophyll a-b binding protein 3, chloroplastic \\
\hline P27521 & CA4_ARATH & Chlorophyll a-b binding protein 4 \\
\hline 082132 & DRE2A_ARATH & Dehydration-responsive element-binding protein $2 \mathrm{~A}$ \\
\hline Q9M0L0 & DRE1A_ARATH & Dehydration-responsive element-binding protein $1 \mathrm{~A}$ \\
\hline P93835 & DRE1B_ARATH & Dehydration-responsive element-binding protein 1B \\
\hline Q9SYS6 & DRE1C_ARATH & Dehydration-responsive element-binding protein $1 \mathrm{C}$ \\
\hline O82132 & DRE2A_ARATH & Dehydration-responsive element-binding protein $2 \mathrm{~A}$ \\
\hline P31168 & COR47_ARATH & Dehydrin COR47 \\
\hline Q94AK4 & RZF1_ARATH & E3 ubiquitin-protein ligase RZF1 \\
\hline Q9M2S6 & SDIR1_ARATH & E3 ubiquitin-protein ligase SDIR1 \\
\hline A0A1S2Z179 & A0A1S2Z179_CICAR & E3 ubiquitin-protein ligase SDIR1-like \\
\hline Q84JL3 & SINA3_ARATH & E3 ubiquitin-protein ligase SINAT3 \\
\hline Q9FNA4 & ELP1_ARATH & Elongator complex protein 1 \\
\hline AOMES8 & ABI4_ARATH & Ethylene-responsive transcription factor $\mathrm{AB} \mid 4$ \\
\hline Q9XI33 & WIN1_ARATH & Ethylene-responsive transcription factor WIN1 \\
\hline P22197 & ALFC7_ARATH & Fructose-bisphosphate aldolase 7 \\
\hline O80518 & GOLS3_ARATH & Galactinol synthase 3 \\
\hline P42761 & GSTFA_ARATH & Glutathione S-transferase F10 \\
\hline Q9ZRW8 & GSTUJ_ARATH & Glutathione S-transferase U19 \\
\hline Q9C9W5 & HPR1_ARATH & Glycerate dehydrogenase HPR, peroxisomal (GDH) \\
\hline Q9M8L4 & GLPK_ARATH & Glycerol kinase (Glycerokinase) \\
\hline Q9LSV0 & GLYR1_ARATH & Glyoxylate/succinic semialdehyde reductase 1 \\
\hline Q9LD83 & SLAC1_ARATH & Guard cell S-type anion channel SLAC1 \\
\hline Q9SXL4 & AHK1_ARATH & Histidine kinase 1 \\
\hline Q9C5U1 & AHK3_ARATH & Histidine kinase 3 \\
\hline Q9C5U0 & AHK4_ARATH & Histidine kinase 4 \\
\hline Q8L9T7 & AHP5_ARATH & Histidine-containing phosphotransfer protein 5 \\
\hline
\end{tabular}


Table 10 (continued)

\begin{tabular}{|c|c|c|}
\hline Entry & Entry name & Protein name \\
\hline Q8VZ59 & YUC6_ARATH & Indole-3-pyruvate monooxygenase YUCCA6 \\
\hline B6UH99 & B6UH99_MAIZE & Late embryogeneis abundant protein Lea14-A \\
\hline Q9M0X3 & Q9M0X3_ARATH & Late embryogenesis abundant \\
\hline F4JQF1 & F4JQF1_ARATH & Late embryogenesis abundant \\
\hline Q9FG31 & LEA46_ARATH & Late embryogenesis abundant protein 46 \\
\hline Q39084 & LEA41_ARATH & Late embryogenis abundant protein \\
\hline Q9XIA9 & LACS2_ARATH & Long chain acyl-CoA synthetase 2 \\
\hline Q95MX3 & VDAC3_ARATH & Mitochondrial outer membrane protein porin 3 \\
\hline O81845 & PUMP1_ARATH & Mitochondrial uncoupling protein 1 (AtPUMP1) \\
\hline Q94A06 & M2K1_ARATH & Mitogen-activated protein kinase kinase 1 \\
\hline O81472 & MP3K2_ARATH & Mitogen-activated protein kinase kinase kinase 9 \\
\hline Q9SQY0 & NAC52_ARATH & NAC domain containing protein 52 \\
\hline Q9XIN7 & NAC40_ARATH & NAC domain-containing protein 40 \\
\hline Q7F2L3 & NAC48_ORYSJ & NAC domain-containing protein 48 \\
\hline Q949N0 & NAC53_ARATH & NAC domain-containing protein 53 \\
\hline Q9SCK6 & NAC62_ARATH & NAC domain-containing protein 62 \\
\hline Q9LD44 & NAC56_ARATH & NAC transcription factor 56 \\
\hline Q0PGJ6 & AKRC9_ARATH & NADPH-dependent aldo-keto reductase \\
\hline Q9SRQ7 & NPC4_ARATH & Non-specific phospholipase C4 \\
\hline Q5U9M2 & Q5U9M2_ORYSJ & Ornithine decarboxylase \\
\hline Q0J265 & Q0J265_ORYSJ & Os09g0375300 protein \\
\hline P24101 & PER33_ARATH & Peroxidase 33 \\
\hline Q9SMU8 & PER34_ARATH & Peroxidase 34 \\
\hline Q8H112 & PGL1A_ARATH & PGR5-like protein 1A \\
\hline Q9FND9 & RFS5_ARATH & Probable galactinol--sucrose galactosyltransferase 5 \\
\hline Q9SJN0 & ABI5_ARATH & Protein ABSCISIC ACID-INSENSITIVE 5 \\
\hline Q9SFB0 & DTX43_ARATH & Protein DETOXIFICATION 43 \\
\hline Q94BS2 & MET1_ARATH & Protein MET1 \\
\hline Q7XJ04 & Q7XJ04_ORYSJ & Putative ornithine decarboxylase \\
\hline Q9LTX3 & PPOX1_ARATH & Pyridoxine/pyridoxamine 5'-phosphate oxidase 1 \\
\hline P22200 & KPYC_SOLTU & Pyruvate kinase, cytosolic isozyme \\
\hline O48791 & SCAB1_ARATH & Stomatal closure-related actin-binding protein 1 \\
\hline 082663 & SDHA1_ARATH & Succinate dehydrogenase flavoprotein subunit 1 \\
\hline Q39232 & SUC1_ARATH & Sucrose transport protein SUC1 \\
\hline Q9LNV3 & STP2_ARATH & Sugar transport protein 2 \\
\hline Q24JK1 & MYB96_ARATH & Transcription factor MYB96 \\
\hline Q9SNC6 & PUB13_ARATH & U-box domain-containing protein 13 \\
\hline Q8RWG1 & AB1K1_ARATH & Protein activity of $\mathrm{BC} 1$ complex kinase 1 \\
\hline Q39096 & ERD15_ARATH & Protein early responsive to dehydration 15 \\
\hline Q9FGI6 & NDUS1_ARATH & NADH dehydrogenase [ubiquinone] iron-sulfur protein 1 \\
\hline Q7XYY2 & MED25_ARATH & Mediator of RNA polymerase II transcription subunit 25 \\
\hline
\end{tabular}

photosynthesis, which would prevent water loss and cell death by attempting to maintain sustainable levels of homeostasis while regulating levels of reactive oxygen species [45].

Two of the seven up-regulated transcript-clusters in M. filifolia leaves were endonucleases. Endonucleases act to remove introns by breaking the phosphodiester bonds to produce functional mRNA [74]. The up-regulation of endonucleases might be degrading mRNA [63]. Messenger RNA degradation prevents the translation of proteins involved in metabolic processes that could cause destabilization under stress. Two proteins 
Table 11 Differentially expressed target genes with a log-fold change (logFC) $\geq 2$ found in each species of Mentzelia and tissue type

\begin{tabular}{|c|c|c|c|c|}
\hline Species/tissue & Target gene & LogFC & $P$-value & Description \\
\hline \multirow[t]{2}{*}{ M. filifolia leaf } & COR47_ARATH & 2.105 & 0.008 & Dehydrin COR47 \\
\hline & ERD15_ARATH & 2.031 & 0.031 & Protein early responsive to dehydration 15 \\
\hline \multirow[t]{2}{*}{ M. filifolia root } & NAC56_ARATH & 2.896 & 0.033 & NAC transcription factor 56 \\
\hline & PGL1A_ARATH & -3.470 & 0.024 & PGR5-like protein 1A \\
\hline \multirow[t]{3}{*}{ M. reverchonii leaf } & NCED3_ARATH & -2.972 & 0.005 & 9-cis-epoxycarotenoid dioxygenase NCED3 \\
\hline & PIP21_ARATH & -3.861 & 0.001 & Aquaporin PIP2-1 \\
\hline & SLAC1_ARATH & -2.648 & 0.019 & Guard cell S-type anion channel SLAC1 \\
\hline \multirow[t]{2}{*}{ M. reverchonii root } & NCED3_ARATH & 3.558 & 0.008 & 9-cis-epoxycarotenoid dioxygenase NCED3 \\
\hline & AUX1_ARATH & -2.109 & 0.053 & Auxin transporter protein 1 \\
\hline M. speciosa leaf & DRE2A_ARATH & 2.209 & 0.031 & Dehydration-responsive element-binding protein $2 \mathrm{~A}$ \\
\hline \multirow[t]{2}{*}{ M. speciosa root } & PYL8_ARATH & -2.532 & 0.033 & Abscisic acid receptor PYL8 \\
\hline & NAC52_ARATH & 2.004 & 0.028 & NAC domain containing protein 5 \\
\hline
\end{tabular}

identified as integral components of the cellular membrane were up-regulated, one specifically identified as TIP1-1 aquaporin protein transmembrane transport, whereas the other is a form of glucosyltransferase involved in the accumulation of the yellow pigment crocetin during fruit development [47]. Aquaporins primarily function to transport water through the cellular membrane, but Zhou et al. [78] determined that the up-regulation of a PIP2 subgroup of aquaporin proteins enhanced drought tolerance in tobacco by increasing the ability to retain water, limit oxidation activity, and decrease the need for antioxidant activity. Aquaporins play an important role in drought stress; however, whether aquaporin proteins are up or down-regulated depends on the species and tissue [59].

Mentzelia reverchonii leaves up-regulated two photosynthetic enzymatic processes (Table 7). Ribulose bisphosphate carboxylase/oxygenase (RuBisCO) was up-regulated, suggesting that photosynthesis signaling increased because it cannot function, or it is deactivated when ratios of ATP/ADP become unfavorable due decreased photosynthesis [12]. The metabolic responses typically associated with drought occur as a response to oxidative stress, rather than a direct response to water deprivation [20]. Because $M$. reverchonii has become accustomed to longer periods of drought stress, its initial response to water deprivation might be to increase carbon assimilation to prepare reserves for stress levels that are intolerable by increasing photosynthetic processes instead of immediately shutting down by reducing gas exchange through stomatal closure.

Mentzelia speciosa leaves responded to drought stress by up-regulating polyubiquitin-like proteins (Table 9). The most common functional role of ubiquitin is the intracellular control of protein content and degradation [66]. Ubiquitin production under stress degrades non-drought stress proteins, which would impair the response to drought stress in an efficient way [66].

\section{Differentially expressed transcript-clusters in roots}

Mentzelia filifolia roots up-regulated cellular respiration, while downregulating the production of secondary metabolites and the oxidation-reduction process (Table 6). When photosynthesis is slowed in the aboveground organs of the plant, carbon is allocated from the root's carbon sinks [26]. Our results based on M. filifolia, therefore, suggests that carbon sequestration in roots is vital to mobilize energy when xerophytes experience drought and photosynthesis is shut down. Although Hasibeder et al. [26] determined that root respiration decreased under prolonged drought conditions in Trisetetum flavescentis, the initial response of down-regulated photosynthesis might result in higher levels of respiration in roots [20]. Up-regulated respiration suggests that recovery efforts might occur to offset the decreased photosynthetic rate [20].

Mentzelia reverchonii roots had a large response to the drought treatment (Table 8). Two of the 54 differentiated transcript-clusters were involved with ubiquitin activity. Increasing the expression of an enzyme responsible for protein degradation would play a direct role in expressed proteins and overall metabolic function [66]. Similar to M. filifolia leaves, auxin transport was up-regulated in a single transcript-cluster, suggesting that another function associated with auxin transport was increased, such as hormonal signaling [54].

Transcript-clusters up-regulated in $M$. reverchonii roots were associated with transmembrane transport, specifically sodium-calcium transmembrane transport 


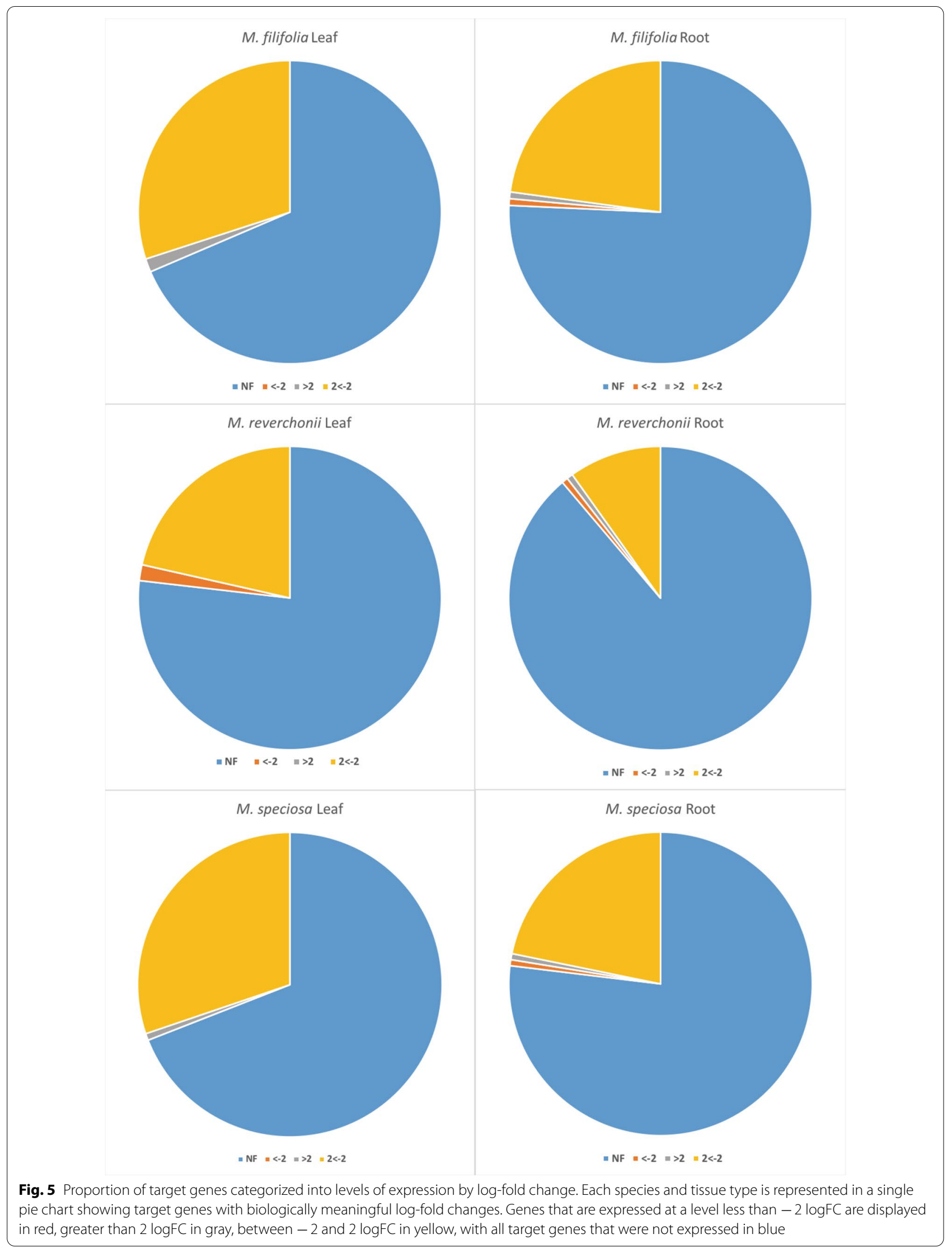


and cellulose synthase. Calcium transport plays a crucial role in drought, salinity stress signaling, and osmoregulation [29]. Zhu et al. [79] determined that cellulose synthase-like proteins in Arabidopsis played a role in osmotic stress tolerance and potentially reactive oxygen species regulation under drought stress. Inositol-tetrakisphosphate regulates the release of intercellular calcium in response to stress [36]. Increasing levels of inositol in Arabidopsis and Solanum greatly increased drought tolerance and decreased abscisic acid levels [36]. One of the largest transcription factor families, no apical meristem (NAC) experienced up-regulation in $M$. reverchonii roots during drought treatment. NAC transcription factors aid in drought tolerance by the regulation of response pathways [69]. Serine/threonine-protein kinase CHK1-like protein, a signal transducer, transcript cluster showed the largest rate of down-regulation in $M$. reverchonii roots. Although CHK1 kinases involved in the DNA damage response (DDR) system have not been identified in plants, a protein of similar function had a large response to drought stress [75]. Plants trigger a DDR system to regulates cell death and DNA repair under stressful conditions [75]. The $M$. reverchonii response might be delaying the need to utilize the DDR to prevent cell death from occurring, similar to M. filifolia leaves delaying senescence.

\section{Similarities in drought tolerance responses through target gene analysis}

Physiological drought responses have a genetic basis [43]. Drought-stress studies on model and crop species have identified common differentially expressed droughtstress genes. Target genes based on previous drought studies were expressed more greatly in M. filifolia and $M$. reverchonii than in $M$. speciosa, further supporting the hypothesis that species adapted to drier environments mount a greater response to drought stress.

Mentzelia filifolia leaves expressed three target genes: Dehydrin COR47, Protein early responsive to dehydration 15, and E3 ubiquitin-protein ligase SDIR1. E3 ubiquitin-protein ligase SDIR1 acts as a positive regulator of abscisic acid stress signal transduction. Improved drought tolerance has been shown in Arabidopsis when over-expression of SIDR1 occurs [76]; however, SDIR1 was under expressed in Mentzelia. Dehydrin COR47 produces a dehydrin hydrophilic protein and was over expressed. Dehydrins accumulate in stressed plant tissues associated with dehydration [30]. Dehydration 15 negatively regulates plant response to abscisic acid [34], and down-regulation of abscisic acid decreases drought tolerance in plants [34]. Our results determined that $M$. filifolia is delaying or down-regulating this particular response to drought.

The target genes NAC-56 and PGR5-like protein 1A were expressed in M. filifolia roots. NAC-56 in root tissues up-regulate target genes that aid in drought tolerance [10]. No apical meristem transcription factors in transgenic-rice roots enhance drought tolerance by targeting genes responsible for changing root architecture [10]. PGR5-like protein 1A, a thylakoid transmembrane protein, was downregulated. PGR5-like protein 1A plays a direct role in the photosynthetic cyclic electron flow that transport electrons to produce ATP [27]. Downregulating the flow of electron transport would decrease or shut down photosynthesis productivity.

Mentzelia reverchonii leaves down-regulated target genes of 9-cis-epoxycarotenoid dioxygenase NCED3, Aquaporin PIP2-1, and Guard cell S-type anion channel SLAC1, while roots up-regulated 9-cis-epoxycarotenoid dioxygenase NCED3 and down-regulated Auxin transporter protein 1 (Table 11), suggesting that the photosynthetic process, or at the very least stomata and transport channels, are being shut down. 9-cis-epoxycarotenoid dioxygenase is a key enzyme in ABA biosynthesis and is induced by drought stress to control the level of endogenous ABA produced [32]. In M. reverchonii, ABA is downregulated in leaves, but upregulated in roots. The down-regulation of plasma membrane intrinsic protein aquaporin might be due to it being a low expression aquaporin when constitutively expressed. The down-regulation of a negative regulator of guard cell anion like the R-type channel that responds rapidly to cystolic $\mathrm{Ca} 2+$ [58] might be what it is utilized for in Mentzelia [64]. An auxin transport protein was down-regulated in the roots of $M$. reverchonii. Down-regulation of molecular and cellular components associated with senescence, like auxin, is regularly down-regulated in Mentzelia, and might be a driving factor in how multiple species tolerate drought stress.

Three target genes were DE in $M$. speciosa leaves and roots (Table 11). Leaves overexpressed Dehydrationresponsive element-binding protein $2 \mathrm{~A}$, which is a gene element that helps regulate expression of genes utilized to cope with drought [52]. Over expression within leaves might be the first response of the cascade of mechanisms plants use to avoid damage from drought stress. A single target gene, NAC domain containing protein 52, was upregulated in M. speciosa. Similar to M. filifolia, NAC-56 in roots is a NAC transcription factor that up-regulates a group of target genes that aid in drought tolerance [10], and the up-regulation of NAC-56 might be to change root architecture to adapt to drought stress [10]. The gene probable pectate lyase 8 (PLY8) was down-regulated within M. speciosa roots. PLY8 is necessary for lateral 
root growth after inhibition by abscisic acid [77]. Downregulation is probably attributed to inhibition through $\mathrm{ABA}$, which is produced in response to drought stress in roots.

\section{Tissue response between species}

Leaves and roots responded differently to drought shock depending on species. When leaves were compared among species, the xeric M. filifolia and semi-arid $M$. reverchonii mounted a stronger response than the mesic M. speciosa (Fig. 4). In contrast, when comparing responses in roots, the xerophytic $M$. filifolia mounted the weakest response compared to the other two species. The genetic response, consequently, appears to be organ and environmental dependent. Although M. speciosa mounted a greater response to drought in roots compared to $M$. filifolia, overall $M$. speciosa responded less to drought shock compared to both $M$. reverchonii and $M$. filifolia.

The differences in tissue response suggests that drought response is tissue, species, and environment specific, and selection pressures related to drought response might be acting on tissues differently. Further research is needed to determine if selection is acting on leaf and root tissues separately. The cascade of physiological response and water regulation described by Bartlett et al. [4] could be tissue specific and instead of studying response as a function of the whole plant, future research should focus on specific tissue responses. Manipulation of genes that play a role in roots or leaves independently might lead to novel pathways for genetic modification allowing for greater drought tolerance that is not only tissue specific but serves to enhance drought tolerance overall.

\section{Conclusions}

Roots and leaves respond to acute drought through different pathways, which are influenced by the environments in which they evolve. Differential expression of genes in response to drought in roots or leaves might lead to novel pathways for genetic modification, allowing for greater drought tolerance that is not only tissue specific, but serves to enhance drought tolerance overall. Delayed senescence played a much larger role than anticipated in both leaves and roots of M. filifolia and $M$. reverchonii. The ability to delay cell death might be a response that has evolved through generations of drought exposure. Risking embolism and desiccation in order to delay senescence is an curious process that should be further studied in xerophytes because it seems to play a major role in their adaptation to drought stress. Mentzelia speciosa produced the weakest response to drought despite having the broadest leaves and occurring in the most mesophytic environment. The individuals of $M$. speciosa had been exposed to less frequent occurrences of drought, potentially leaving them without the evolved ability to respond in an efficient and timely way. We also observed differences in drought response depending on tissue type suggesting that species could have a mounted response within one tissue type and not the other, and tissue-specific responses could be evolving at different rates. Further studies that utilize quantitative qPCR would help to verify the presence of target genes within tissues and the magnitude of the regulation taking place. Our results suggest that, in additional to morphological evolution to limit drought stress, xerophytes have evolved a cascade of genetic responses that have tissue-specific responses to mitigate drought stress through delayed senescence, decreased photosynthesis, and decreased water transport.

\section{Methods}

Species of Mentzelia occur across a wide environmental gradient, from southwestern North American deserts, to mesic habitats near the Continental Divide in the Rocky Mountains [60]. Despite their ecological importance across western North America, and especially in drought-prone gypsum outcrops [60, 61], we do not understand the mechanisms behind their drought tolerance and success in xeric habitats. Three species of Mentzelia were sampled that occur across an environmental gradient, from desert to mesic ecosystems (Fig. 6). The xerophytic M. filifolia was sampled in the New Mexican Chihuahuan Desert. Mentzelia reverchonii, a semi-arid species, was collected in the Texas short-grass prairies. The mesophytic M. speciosa was sampled in the central Rocky Mountains of Colorado. Natural populations of all three species were sampled because plants failed to grow in greenhouse conditions. The three sampled species belong to the same section within Mentzelia, section Bartonia, but are not each other's closest relatives [62]. At the time of collection, the M. filifolia population in New Mexico received $2.16 \mathrm{~cm}$ of precipitation during July and has a mean annual precipitation of $19.71 \mathrm{~cm} \mathrm{[16].}$ The $M$. reverchonii population in Texas received $4.24 \mathrm{~cm}$ of precipitation for June, with a mean annual precipitation of $53.19 \mathrm{~cm}$ [16]. The M. speciosa population in Colorado received $3.3 \mathrm{~cm}$ of precipitation in July, and has a mean annual precipitation of $55.60 \mathrm{~cm}$ [16]. All habitats experienced average rates of rainfall for July according to precipitation data from the past 30 years.

\section{Field sampling}

Natural populations were sampled on separate dates in the months of June and July, 2017. Mentzelia filifolia was collected northwest of Gallup, New Mexico on July 12th, 2017 (35.65186 $\left.\mathrm{N}, 109.02622^{\circ} \mathrm{W}, 2080 \mathrm{~m}\right)$. Sampling 


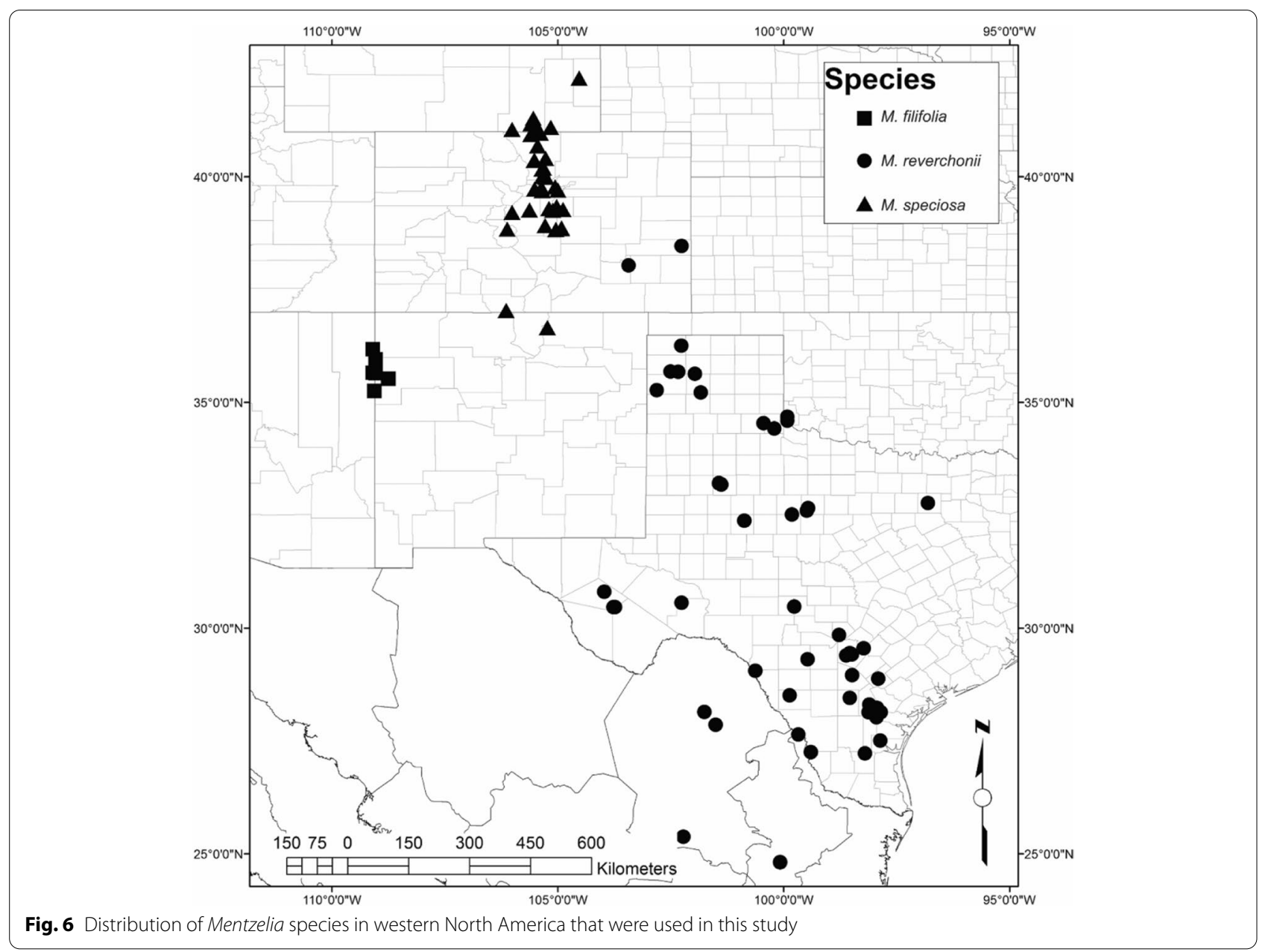

occurred at 5:00 PM with a temperature of $31^{\circ} \mathrm{C}$. Individuals of $M$. reverchonii were collected in Shackelford County, near Fort Phantom Lake in Abilene, Texas on June 27th, $2017\left(32.606747^{\circ} \mathrm{N}, 99.692199^{\circ} \mathrm{W}, 511 \mathrm{~m}\right)$. Sampling occurred at 6:00 PM with a temperature of $32^{\circ} \mathrm{C}$. Individuals of $M$. speciosa were collected southwest of Lyons, Colorado on July 5th, 2017 (40. 202,972 $\mathrm{N}$ $-105.299625^{\circ} \mathrm{W}, 1900 \mathrm{~m}$ ). Sampling occurred at 2:00 PM with a temperature of $32^{\circ} \mathrm{C}$. Four control and four treatment plants that were developmentally identical (bolted from the rosette stage with flowers present) were selected randomly while maximizing distance between them to avoid sampling closely related individuals. Four treatment plants were excavated with their roots intact and placed on the ground in full sun. We refer to this approach as a drought-shock treatment, which has been used in other studies to examine the response to drought in natural populations (e.g., $[48,56])$. While the drought-shock treatment was being conducted, four control plants were excavated with roots intact and sampled immediately to avoid sampling drought-stressed tissues. Control plants were excavated prior to leaf sampling to ensure that any wound response associated with the extraction from the ground would be identified in both the control and treatment plants, which would result in no differentially expressed (DE) genes associated with wounding after applying our bioinformatics pipeline (see below). Leaf and root tissues were collected in replicates of three, while flower, fruit, and stem tissues were collected from one individual per population for the purpose of generating a reference transcriptome. Mature leaf tissues with no insect damage were immediately placed in $1.5 \mathrm{~mL}$ of RNAlater ${ }^{\mathrm{TM}}$ RNA Stabilization Solution (Thermo Fisher Scientific, Waltham, Massachusetts, U.S.A.) to preserve the RNA. Root tissues were sampled after the leaf tissues were sampled. Excess soil was removed by brushing off the roots, but much of the remaining soil washed away before RNA extraction. Approximately $100 \mathrm{~g}$ of tissue from the middle of the central tap root was sampled. The treatment plants were subjected to an hour of full sun exposure until leaves began to wilt and curl, indicating that the plants were experiencing acute drought 
stress. All tissue samples were placed in a $-80^{\circ} \mathrm{C}$ freezer approximately 3-5days after collection until the RNA was extracted. Vouchers for the collected population were pressed and deposited in the Georgia Southern University Herbarium (GAS).

\section{RNA extraction}

Tissue samples were thawed, removed from RNAlater, and placed into new tubes with $2.8 \mathrm{~mm}$ ceramic beads. Samples were frozen and homogenized with a Qiagen Tissuelyser II (Qiagen, Hilden, Germany) for $1 \mathrm{~min}$ at $30 \mathrm{~Hz}$. TRIzol extraction buffer (Thermo Fisher Scientific) was added in aliquots of $1 \mathrm{~mL}$ to each sample, homogenized for nine additional minutes, and then incubated for $5 \mathrm{~min}$ at room temperature. The phase separator, 1-bromo-3-chloropropane, was added in $100 \mu \mathrm{l}$ aliquots, vortexed for $15 \mathrm{~s}$, and incubated at room temperature for $5 \mathrm{~min}$. Samples were centrifuged at 12,000 g for $10 \mathrm{~min}$. The upper aqueous phase was transferred to a new tube and $500 \mu \mathrm{l}$ of chilled isopropanol was added. The samples were stored overnight in a $-80^{\circ} \mathrm{C}$ freezer. After approximately $14 \mathrm{~h}$, the RNA samples were removed from the freezer and centrifuged at $12,000 \mathrm{~g}$ for $10 \mathrm{~min}$. The supernatant was discarded, $1 \mathrm{~mL}$ of chilled $75 \% \mathrm{EtOH}$ was added, and samples were centrifuged for $5 \mathrm{~min}$ at $12,000 \mathrm{~g}$. All supernatant was removed, and samples were air dried in a fume hood for $30 \mathrm{~min}$. The RNA was resuspended into $60 \mu \mathrm{l}$ of nuclease-free water. The concentration of each RNA sample was quantified using both a Qubit Fluorometer (Qubit 2.0; Invitrogen, Life Technologies, California, U.S.A.) and an Agilent 2100 Bioanalyzer (Agilent Technologies, Inc., Waldbronn, Germany).

\section{cDNA library creation}

cDNA libraries were created from each RNA isolation. We used the NEBNext Ultra RNA Library Prep Kit for Illumina (New England Biolabs, Ipswich, Massachusetts, U.S.A.) in conjunction with the NEBNext Poly(A) mRNA Magnetic Isolation Module (New England Biolabs) and the NEBNext Multiplex Oligos for Illumina (New England Biolabs). All libraries were generated following the manufacturer's protocol. Both the Qubit Fluorometer (Qubit 2.0 HS DNA assay) and the Agilent 2100 Bioanalyzer were used to quantify each library. All cDNA libraries were pooled together to maintain a $10 \mathrm{mM}$ concentration, and then sequenced on an Illumina NextSeq (150 Cycles) PE75 High Output flow cell at the Georgia Genomics and Bioinformatics Core at the University of Georgia.
Reference transcriptome generation, annotation, and comparisons

Because no reference transcriptome is published for Mentzelia, we generated de novo transcriptome assemblies that were applied as references in subsequent analyses. Raw-sequence read-quality was assessed with the FASTX-Toolkit [22]. Reads were quality filtered and trimmed in Trimmomatic v0.36 [7] to remove adapter sequences, ambiguous nucleotides, low quality sequences with Phred scores $\leq 20$, and sequences $<36$ bp in length [42]. Flower, fruit, stressed and unstressed roots, stressed and unstressed leaves, and stem tissue sequences were combined to generate two separate reference transcriptomes of $M$. speciosa and M. filifolia. Sequence reads were assembled in Trinity v2.4.0 [25] for de novo generation [23] with a K-mer size of 25, which is a sufficient size for a de novo assembly for a non-model organism with no reference genome [24].

To ensure that each transcriptome was complete and an adequate representation of both species, HISAT2 v2.0.5 [51] was used to map back the trimmed sequence reads from each sample. Average alignment percentages were calculated to ensure at least an $80 \%$ alignment rate average.

Trinotate (https://trinotate.github.io/) was used for the comprehensive de novo transcriptome annotation. Trinotate utilizes BLAST [1] and SwissProt [6] to infer homology based on sequence similarity, HMMER [19] and PFAM [3] for protein domain identification, and eggNOG [31], GO [2, 8, 21], and KEGG databases [33] to identify functional groups or pathways. All programs were used in conjunction with Trinotate to create a functional annotation for each transcriptome generated using the output from each search to populate an SQlite database [70]. The SQlite database was used to create an annotation report showing all results from each respective database search.

HISAT2 [51] was used to assess the completeness and quality of the transcriptome alignments by re-mapping the trimmed reads back to the transcriptome alignment. A BUSCO [67] analysis was performed on the remapped reads to determine the completeness of each de novo assembled transcriptome by comparing present singlecopy orthologs using the provided eukaryotic lineage dataset to the generated annotated assembly.

\section{Expression analysis}

Burrows-Wheeler Aligner (BWA) v0.7.13 [39] determined transcript level abundance by mapping low divergence transcript sequences to a reference transcriptome. We employed the M. filifolia and $M$. speciosa reference transcriptomes to conduct reference-guided assemblies. Reference transcriptomes for the respective species were 
used as the target inputs, using the reference from the closely related M. speciosa for M. reverchonii [62]. Each transcriptome was made into an FM-index to compress full text files for faster alignment rates. After the indices were made, the options for a mismatch penalty of 0.05 , a gap open penalty of one, no output lower than 10, 20 threads, and "mem" option for local alignment of transcripts back to the reference were used for BWA analysis. SAMtools v1.3 [40] was used to convert the SAM output files from BWA to sorted BAM files, which were inputted into Corset v1.07 [13] that hierarchically grouped transcript contigs into clusters by shared reads and expression data. Counts of the number of transcripts included in each cluster were made and used as the input raw count data for differential expression analyses. The edgeR package $[44,57]$ was used for differential expression analysis in R [53]. Transcript-cluster count files were read in by species and tissue type separately (e.g., root tissues of $M$. speciosa), with individuals grouped together by control or treatment. The DGEList function was used to create an object from the transcript cluster-counts for each species and tissue type individually. Transcript clusters with fewer than one transcript count per million in fewer than six of the eight individuals were discarded to reduce the number of rarely expressed genes that were not DE across all members of a group. Normalized factors were calculated to scale each library size. Common dispersion was calculated to maximize the negative binomial, conditional common-likelihood to estimate a common dispersion value across all genes. Tagwise dispersion was estimated with an empirical Bayes method based on weighted conditional maximum likelihood [44, 57]. We used the exact test to determine differences in mean values between the two negative binomially distributed counts. A false discovery rate $P$-value adjustment was used to address multiple comparisons. We also compared DE clusters with log-fold changes of two or greater. Differentially expressed clusters were annotated with Blast2Go [11] to identify their gene or protein name, along with a description and function.

Bivariate plots were generated in $\mathrm{R}$, and differentially expressed genes with a log-fold values of 2 or greater were indicated. Differential expression values were estimated by comparing the treatment to the control for each species. A slope of one is expected if expression levels between species are identical, and we tested whether expression significantly deviated with the one-sample slope-test with the smatr package [71] in R. Statistical tests were conducted by constructing distributions for the test statistic following Taskinen and Warton [68].

\section{Target gene approach}

Complementary to the transcriptome profiling approach, we applied a target gene approach to search for expression patterns in drought associated genes. In comparison to approaches that strictly profile the transcriptome for significantly DE genes, a targeted approach has the ability to determine the exact levels of differential expression [43] and whether genes associated with drought response are expressed at all. The target approach can determine commonalities in stress response across plants, allowing us to identify genes that commonly or uniquely respond to drought. We took advantage of previous studies to create a list of drought-tolerant genes and their sequences, then explicitly measured the response of the targeted genes to determine if Mentzelia responded similarly to drought stress.

We selected 90 genes from different gene families known to play a role in drought response (e.g., $[9,35,38$, $41,46,49,50,65,78])$, as well as genes with GO terms related to or associated with drought tolerance from GenBank [5]. The target genes from Arabidopsis, corn, soy bean, and sorghum were downloaded from GenBank. The entry name for each target gene was searched within sequence annotation reports from the reference transcriptomes to identify the associated transcript-cluster's ID. The edgeR results from the differential expression exact test for each species and tissue type was searched using the annotated genes or protein names to identify if the associated gene clusters and the level of gene expression for each target gene.

\section{Abbreviations}

ABA: Abscisic acid; ATP: Denosine triphosphate; DE: Differential expression; DDR: DNA damage response; FDR: False discovery rate; HA-1: Alternative hypothesis 1; HA-2: Alternative hypothesis 2; HA-3: Alternative hypothesis 3; Ho: Null hypothesis; logFC: Log-fold change; mRNA: Messenger RNA; NAC: No apical meristem; rDNA: Ribosomal DNA; RuBisCO: Ribulose bisphosphate carboxylase/oxygenase.

\section{Supplementary Information}

The online version contains supplementary material available at https://doi. org/10.1186/s12864-021-08178-w.

Additional file 1 : Supplemental Data. Log-fold change in expression levels of target genes found in the results of the exact test to determine differential expression for each tissue type, root and leaf, and all three species; Mentzelia filifolia, M. reverchonii, and M. speciosa. Values for log-fold changes (LogFC) show the direction and extent of the change of expression for each target gene. Values of LogFC with * indicate values with corresponding significant $p$-values $<0.05$.

\section{Acknowledgements}

We are grateful to Jim Long and John David Curlis who helped with field sampling and analysis. We thank Max Perkins for helping with the bioinformatics 
analysis, Josh Brokaw for providing sampling locations, and Christian Cox for helpful comments.

\section{Authors' contributions}

JD performed field sampling, all wet lab methodology, data analysis and transcriptome generation and annotation, and was the lead writer of the manuscript. AC performed script writing for the bioinformatics workflow for transcriptome generation and expression analysis. JJS helped with experimental design and field sampling, performed data analysis, and was a main contributor on the manuscript. All authors read and approved the final manuscript.

\section{Funding}

This project was funded by the Georgia Southern University Graduate Student Organization and the GSU College of Science and Mathematics.

\section{Availability of data and materials}

The datasets generated and/or analyzed during the current study are available in the NCBI GenBank repository: ncbi.nlm.nih.gov/genbank/. Raw sequence reads and assembled transcriptome data have been deposited under the BioProject accession number: PRJNA708198.

\section{Declarations}

\section{Ethics approval and consent to participate}

Not applicable.

\section{Consent for publication}

Not applicable.

\section{Competing interests}

The authors declare that they have no competing interests.

\section{Author details}

1Department of Biology, Georgia Southern University, Statesboro, GA 30460, USA. ${ }^{2}$ Department of Ecology and Evolutionary Biology, University of California Los Angeles, Los Angeles, CA 90095-7246, USA. ${ }^{3}$ Department of Environmental and Plant Biology, Ohio University, Athens, OH 457012979, USA.

Received: 1 December 2020 Accepted: 16 November 2021 Published online: 04 January 2022

\section{References}

1. Altschul SF, Gish W, Miller W, Myers EW, Lipman DJ. Basic local alignment search tool. J Mol Biol. 1990;215(3):403-10.

2. Ashburner M, Ball CA, Blake JA, Botstein D, Butler H, Cherry JM, et al. Gene ontology: tool for the unification of biology. Nat Genet. 2000;25(1):25-9.

3. Bateman A, Coin L, Durbin R, Finn RD, Hollich V, Griffiths-Jones $\mathrm{S}$, et al. The Pfam protein families database. Nucleic Acids Res. 2004;32(suppl_1):D138-41.

4. Bartlett MK, Klein T, Jansen S, Choat B, Sack L. The correlations and sequence of plant stomatal, hydraulic, and wilting responses to drought. PNAS. 2016:113:13098-103.

5. Benson DA, Karsch-Mizrachi I, Lipman DJ, Ostell J, Wheeler DL. GenBank Nucleic Acids Res. 2005:33:D34-8.

6. Boeckmann B, Bairoch A, Apweiler R, Blatter MC, Estreicher A, Gasteiger E, et al. The SWISS-PROT protein knowledgebase and its supplement TrEMBL in 2003. Nucleic Acids Res. 2003;31(1):365-70.

7. Bolger AM, Lohse M, Usadel B. Trimmomatic: a flexible trimmer for Illumina sequence data. Bioinformatics. 2014:30:2114-20.

8. Carbon S, Ireland A, Mungall CJ, Shu S, Marshall B, Lewis S. AmiGO hub, web presence working group. AmiGO: online access to ontology and annotation data. Bioinformatics. 2009;25(2):288-9.

9. Chen Q, Niu F, Yan J, Chen B, Wu F, Guo X, et al. Oilseed rape NAC56 transcription factor modulates reactive oxygen species accumulation and hypersensitive response-like cell death. Physiol Plant. 2017;160:209-21.
10. Chung PJ, Jung H, Do Choi Y, Kim JK. Genome-wide analyses of direct target genes of four rice NAC-domain transcription factors involved in drought tolerance. BMC Genomics. 2018;19:40.

11. Conesa A, Götz S. Blast2GO: a comprehensive suite for functional analysis in plant genomics. Int J Plant Genomics. 2008;2008:619832.

12. Crafts-Brandner SJ, Salvucci ME. Rubisco activase constrains the photosynthetic potential of leaves at high temperature and CO2. PNAS. 2000;97:13430-5

13. Davidson NM, Oshlack A. Corset: enabling differential gene expression analysis for de novo assembled transcriptomes. Genome Biol. 2014;15:1-4

14. DeBiasse MB, Kelly MW. Plastic and evolved responses to global change: what can we learn from comparative transcriptomics? J Hered. 2016;107:71-81.

15. Drake-Schultheis $L$, Oono R, D'Antonio CM. Mechanisms of severe dieback and mortality in a classically drought-tolerant shrubland species (Arctostaphylos glauca). Am J Bot. 2020;107:1136-47.

16. Eischeid JK, Bruce Baker C, Karl TR, Diaz HF. The quality control of longterm climatological data using objective data analysis. J Appl Meterol. 1995:34:2787-95

17. El Hafid R, Smith DH, Karrou M, Samir K. Physiological responses of spring durum wheat cultivars to early-season drought in a Mediterranean environment. Ann Bot. 1998;81:363-70.

18. Farooq M, Wahid A, Kobayashi N, Fujita DB, Basra SM. Plant drought stress: effects, mechanisms and management. Sustain Agric. 2009:29:153-88.

19. Finn RD, Clements J, Eddy SR. HMMER web server: interactive sequence similarity searching. Nucleic Acids Res. 2011;39(suppl_2):W29-37.

20. Flexas J, Bota J, Galmes J, Medrano H, Ribas-Carbó M. Keeping a positive carbon balance under adverse conditions: responses of photosynthesis and respiration to water stress. Physiol Plant. 2006;127:343-52.

21. Gene Ontology Consortium. The gene ontology project in 2008. Nucleic Acids Res. 2008:36(suppl 1):D440-4

22. Gordon A, Hannon GJ. Fastx-toolkit. FASTQ/a short-reads preprocessing tools. 2010. http://hannonlab.cshl.edu/fastx_toolkit.

23. Grabherr MG, Haas BJ, Yassour M, Levin JZ, Thompson DA, Amit I, et al. Full-length transcriptome assembly from RNA-Seq data without a reference genome. Nat Biotechnol. 2011;29:644-52.

24. Grizante MB, Tollis M, Rodriguez JJ, Levy O, Angilletta MJ, Kusumi K. De novo assembly and annotation of the eastern fence lizard (Sceloporus undulatus) transcriptome. BioRxiv. 2017:136069.

25. Haas BJ, Papanicolaou A, Yassour M, Grabherr M, Blood PD, Bowden J, et al. De novo transcript sequence reconstruction from RNA-seq using the trinity platform for reference generation and analysis. Nat Protoc. 2013;8:1494-512.

26. Hasibeder R, Fuchslueger L, Richter A, Bahn M. Summer drought alters carbon allocation to roots and root respiration in mountain grassland. New Phytol. 2015;205:1117-27.

27. Hertle AP, Blunder T, Wunder T, Pesaresi P, Pribil M, Armbruster U, et al. PGRL1 is the elusive ferredoxin-plastoquinone reductase in photosynthetic cyclic electron flow. Mol Cell. 2013:49:511-23.

28. Hoover DL, Duniway MC, Belnap J. Testing the apparent resistance of three dominant plants to chronic drought on the Colorado plateau. J Ecol. 2017;105:152-62.

29. Hu Y, Schmidhalter U. Drought and salinity: a comparison of their effects on mineral nutrition of plants. J Plant Nutr Soil Sci. 2005;168:541-9.

30. Hu L, Wang Z, Du H, Huang B. Differential accumulation of dehydrins in response to water stress for hybrid and common bermudagrass genotypes differing in drought tolerance. J Plant Physiol. 2010;167:103-9.

31. Huerta-Cepas J, Szklarczyk D, Forslund K, Cook H, Heller D, Walter MC et al. eggNOG 4.5: a hierarchical orthology framework with improved functional annotations for eukaryotic, prokaryotic and viral sequences. Nucleic Acids Res. 2016;44(D1):D286-93.

32. Iuchi S, Kobayashi M, Taji T, Naramoto M, Seki M, Kato T, et al. Regulation of drought tolerance by gene manipulation of 9-cis-epoxycarotenoid dioxygenase, a key enzyme in abscisic acid biosynthesis in Arabidopsis. Plant J. 2001;27:325-33.

33. Kanehisa M, Goto S. KEGG: Kyoto encyclopedia of genes and genomes. Nucleic Acids Res. 2000;28(1):27-30.

34. Kariola T, Brader G, Helenius E, Li J, Heino P, Palva ET. Early responsive to dehydration 15, a negative regulator of abscisic acid responses in Arabidopsis. Plant Physiol. 2006;142:1559-73. 
35. Ke Q, Wang Z, Ji CY, Jeong JC, Lee HS, Li H, et al. Transgenic poplar expressing Arabidopsis YUCCA6 exhibits auxin-overproduction phenotypes and increased tolerance to abiotic stress. Plant Physiol Biochem. 2015;94:19-27.

36. Khodakovskaya M, Sword C, Wu Q, Perera IY, Boss WF, Brown CS, et al. Increasing inositol $(1,4,5)$-trisphosphate metabolism affects drought tolerance, carbohydrate metabolism and phosphate-sensitive biomass increases in tomato. Plant Biotechnol J. 2010;8:170-83.

37. Koo YJ, Yoon ES, Seo JS, Kim JK, Do CY. Characterization of a methyl jasmonate specific esterase in Arabidopsis. J Korean Soc Appl Bio Chem. 2013;56:27-33.

38. Le DT, Nishiyama RI, Watanabe Y, Mochida K, Yamaguchi-Shinozaki K, Shinozaki K, et al. Genome-wide expression profiling of soybean twocomponent system genes in soybean root and shoot tissues under dehydration stress. DNA Res. 2011;18:17-29.

39. Li H, Durbin R. Fast and accurate short read alignment with burrowsWheeler transform. Bioinformatics. 2009;25:1754-60.

40. Li H, Handsaker B, Wysoker A, Fennell T, Ruan J, Homer N, et al. The sequence alignment/map format and SAMtools. Bioinformatics. 2009;25:2078-9.

41. Li C, Nong Q, Solanki MK, Liang Q, Xie J, Liu X, et al. Differential expression profiles and pathways of genes in sugarcane leaf at elongation stage in response to drought stress. Sci Rep. 2016;6:25698.

42. Ma J, Li R, Wang H, Li D, Wang X, Zhang Y, et al. Transcriptomics analyses reveal wheat responses to drought stress during reproductive stages under field conditions. Front Plant Sci. 2017;8:592.

43. Marra NJ, Eo SH, Hale MC, Waser PM, DeWoody JA. A priori and a posteriori approaches for finding genes of evolutionary interest in non-model species: Osmoregulatory genes in the kidney transcriptome of the desert rodent Dipodomys spectabilis (banner-tailed kangaroo rat). Comp Biochem Physiol Part D Genomics Proteomics. 2012;7:328-39.

44. McCarthy DJ, Chen Y, Smyth GK. Differential expression analysis of multifactor RNA-Seq experiments with respect to biological variation. Nucleic Acids Res. 2012;40:4288-97.

45. Miller GA, Suzuki N, Ciftci-Yilmaz SU, Mittler RO. Reactive oxygen species homeostasis and signaling during drought and salinity stresses. Plant Cell Environ. 2010;33:453-67.

46. Muthuramalingam P, Krishnan SR, Pothiraj R, Ramesh M. Global transcriptome analysis of combined abiotic stress signaling genes unravels key players in Oryza sativa L.: an in silico approach. Front. Plant Sci. 2017;8:759.

47. Nagatoshi M, Terasaka K, Owaki M, Sota M, Inukai T, Nagatsu A, et al. UGT75L6 and UGT94E5 mediate sequential glucosylation of crocetin to crocin in Gardenia jasminoides. FEBS Lett. 2012;586:1055-61.

48. Nguyen $\mathrm{KH}, \mathrm{Ha}$ CV, Watanabe Y, Tran UT, Nasr Esfahani M, Nguyen DV, et al. Correlation between differential drought tolerability of two contrasting drought-responsive chickpea cultivars and differential expression of a subset of CaNAC genes under normal and dehydration conditions. Front Plant Sci. 2015;6:449.

49. Osakabe Y, Osakabe K, Shinozaki K, Tran LS. Response of plants to water stress. Front Plant Sci. 2014;5:86.

50. Pan L, Meng C, Wang J, Ma X, Fan X, Yang Z, et al. Integrated omics data of two annual ryegrass (Lolium multiflorum L.) genotypes reveals core metabolic processes under drought stress. BMC Plant Biol. 2018;18:26.

51. Pertea M, Kim D, Pertea GM, Leek JT, Salzberg SL. Transcript-level expression analysis of RNA-seq experiments with HISAT, StringTie and Ballgown. Nat Protoc. 2016;11:1650.

52. Qin F, Sakuma Y, Tran LS, Maruyama K, Kidokoro S, Fujita Y, et al. Arabidopsis DREB2A-interacting proteins function as RING E3 ligases and negatively regulate plant drought stress-responsive gene expression. Plant Cell. 2008;20:1693-707.

53. R Development Core Team. R: a language and environment for statistical computing. 2005; http://cranr-project.org.

54. Rahman A. Auxin: a regulator of cold stress response. Physiol Plant. 2013;147(1):28-35.

55. Rivero RM, Kojima M, Gepstein A, Sakakibara H, Mittler R, Gepstein S, et al. Delayed leaf senescence induces extreme drought tolerance in a flowering plant. PNAS. 2007;104:19631-6.

56. Rizhsky L, Liang H, Mittler R. The combined effect of drought stress and heat shock on gene expression in tobacco. Plant Physiol. 2002;130:1143-51.
57. Robinson MD, McCarthy DJ, Smyth GK. edgeR: a Bioconductor package for differential expression analysis of digital gene expression data. Bioinformatics. 2010;26:139-40.

58. Sah SK, Reddy KR, Li J. Abscisic acid and abiotic stress tolerance in crop plants. Front Plant Sci. 2016;7:571.

59. Santoni V, Vinh J, Pflieger D, Sommerer N, Maurel C. A proteomic study reveals novel insights into the diversity of aquaporin forms expressed in the plasma membrane of plant roots. Biochem J. 2003;373:289-96.

60. Schenk JJ. Biogeographical diversification of Mentzelia section Bartonia in western North America. J Biogeogr. 2013a;40:455-65.

61. Schenk JJ. Evolution of limited seed dispersal ability on gypsum islands. Am J Bot. 2013b;100:1811-22.

62. Schenk JJ, Hufford L. Phylogeny and taxonomy of Mentzelia section Bartonia (Loasaceae). Sys Bot. 2011;36:711-20.

63. Schoenberg DR. Mechanisms of endonuclease-mediated mRNA decay. Wiley Interdiscip Rev RNA. 2011;2:582-600.

64. Schroeder J, Keller BU. Two types of anion channel currents in guard cells with distinct voltage regulation. PNAS. 1992;89:5025-9.

65. Seki M, Narusaka M, Ishida J, Nanjo T, Fujita M, Oono Y, et al. Monitoring the expression profiles of 7000 Arabidopsis genes under drought, cold and high-salinity stresses using a full-length cDNA microarray. Plant J. 2002;31:279-92.

66. Sharma B, Joshi D, Yadav PK, Gupta AK, Bhatt TK. Role of ubiquitin-mediated degradation system in plant biology. Front Plant Sci. 2016;7:806.

67. Simão FA, Waterhouse RM, loannidis P, Kriventseva EV, Zdobnov EM. BUSCO: assessing genome assembly and annotation completeness with single-copy orthologs. Bioinformatics. 2015;31(19):3210-2.

68. Taskinen S, Warton DI. Robust estimation and inference for bivariate linefitting in allometry. Biom J. 2011;53:652-72.

69. Thao NP, Thu NB, Hoang XL, Ha CV, Tran LS. Differential expression analysis of a subset of drought-responsive GmNAC genes in two soybean cultivars differing in drought tolerance. Int J Mol Sci. 2013;14:23828-41.

70. Thunders M, Cavanagh J, Li Y. De novo transcriptome assembly, functional annotation and differential gene expression analysis of juvenile and adult E. fetida, a model oligochaete used in ecotoxicological studies. Biol Res. 2017;50(1):1-5.

71. Warton DI, Duursma RA, Falster DS, Taskinen S. Smatr 3-an R package for estimation and inference about allometric lines. Methods Ecol and Evol. 2012;2012(3):257-9

72. Wei C, Tao X, Li M, He B, Yan L, Tan X, et al. De novo transcriptome assembly of Ipomoea nil using Illumina sequencing for gene discovery and SSR marker identification. Mol Genet Genomics. 2015:290:1873-84.

73. $\mathrm{Xu} \mathrm{Z,} \mathrm{Zhou} \mathrm{G,} \mathrm{Shimizu} \mathrm{H.} \mathrm{Plant} \mathrm{responses} \mathrm{to} \mathrm{drought} \mathrm{and} \mathrm{rewatering.}$ Plant Signal Behav. 2010;5:649-54.

74. Xue S, Calvin K, Li H. RNA recognition and cleavage by a splicing endonuclease. Science. 2006;312:906-10.

75. Yoshiyama KO, Kimura S, Maki H, Britt AB, Umeda M. The role of SOG1, a plant-specific transcriptional regulator, in the DNA damage response. Plant Signal Behav. 2014;9:e28889.

76. Zhang YY, Li Y, Gao T, Zhu H, Wang DJ, Zhang HW, et al. Arabidopsis SDIR1 enhances drought tolerance in crop plants. Biosci Biotechnol Biochem. 2008;72:2251-4.

77. Zhao Y, Xing L, Wang X, Hou YJ, Gao J, Wang $P$, et al. The ABA receptor PYL8 promotes lateral root growth by enhancing MYB77-dependent transcription of auxin-responsive genes. Sci Signal. 2014;7:ra53.

78. Zhou S, Hu W, Deng X, Ma Z, Chen L, Huang C, et al. Overexpression of the wheat aquaporin gene, TaAQP7, enhances drought tolerance in transgenic tobacco. PLoS One. 2012;7:e52439.

79. Zhu J, Lee BH, Dellinger M, Cui X, Zhang C, Wu S, et al. A cellulose synthase-like protein is required for osmotic stress tolerance in Arabidopsis. Plant J. 2010;63:128-40.

\section{Publisher's Note}

Springer Nature remains neutral with regard to jurisdictional claims in published maps and institutional affiliations. 